Les rapports sociaux de sexe, les appartenances de classe et de race et la réussite en enseignement postsecondaire : l'état de la question

\author{
Social Interaction of the Sexes, Class and Race Affiliations, and \\ Success in Higher Education: State of the Art Report \\ Las relaciones sociales de sexo, las membresías de clase y de \\ raza y el éxito en enseñanza post-secundaria: balance de la \\ cuestión
}

\title{
Renée Cloutier
}

Volume 33, numéro 1, printemps 2005

Les femmes en éducation et en formation

URI : https://id.erudit.org/iderudit/1079068ar

DOI : https://doi.org/10.7202/1079068ar

\section{Aller au sommaire du numéro}

\section{Éditeur(s)}

Association canadienne d'éducation de langue française

\section{ISSN}

0849-1089 (imprimé)

1916-8659 (numérique)

Découvrir la revue

Citer cet article

Cloutier, R. (2005). Les rapports sociaux de sexe, les appartenances de classe et de race et la réussite en enseignement postsecondaire : l'état de la question. Éducation et francophonie, 33(1), 165-214. https://doi.org/10.7202/1079068ar

\section{Résumé de l'article}

Les femmes étant majoritaires aux études collégiales et au premier cycle universitaire aux États-Unis, en Angleterre et au Canada, on pourrait penser que l'appartenance sexuelle n'est plus considérée comme un des enjeux majeurs des études sur la réussite éducative en enseignement postsecondaire (higher education). Ce phénomène s'observe-t-il dans les études sur ce thème publiées dans les écrits scientifiques en sciences sociales appliquées à l'éducation en 2002? La progression des femmes fait-elle en sorte que les chercheurs et les chercheuses leur accordent moins d'attention au profit d'autres groupes sociaux? Les rapports sociaux de sexe constituent-ils l'objet central ou périphérique de ces recherches? L’appartenance sexuelle est-elle traitée de concert avec d'autres types d'appartenance, comme la classe sociale, la race ou l'ethnie? Les cadres d'analyse féministe font-ils partie des approches théoriques? Que nous apprennent ces études? Peut-on formuler l'hypothèse du maintien ou de la progression des femmes aux études postsecondaires dans les pays industrialisés au cours des années à venir?
Tous droits réservés (C) Association canadienne d'éducation de langue française, 2005
Cecument est protégé par la loi sur le droit d'auteur. L'utilisation des services d'Érudit (y compris la reproduction) est assujettie à sa politique d'utilisation que vous pouvez consulter en ligne.

https://apropos.erudit.org/fr/usagers/politique-dutilisation/ 


\section{Les rapports sociaux de sexe, les appartenances de classe et de race et la réussite en enseignement postsecondaire : l'état de la question}

\section{Renée CLOUTIER}

Faculté des sciences de l'éducation, Université Laval, Canada

\section{RÉSUMÉ}

Les femmes étant majoritaires aux études collégiales et au premier cycle universitaire aux États-Unis, en Angleterre et au Canada, on pourrait penser que l'appartenance sexuelle n'est plus considérée comme un des enjeux majeurs des études sur la réussite éducative en enseignement postsecondaire (higher education). Ce phénomène s'observe-t-il dans les études sur ce thème publiées dans les écrits scientifiques en sciences sociales appliquées à l'éducation en 2002? La progression des femmes fait-elle en sorte que les chercheurs et les chercheuses leur accordent moins d'attention au profit d'autres groupes sociaux? Les rapports sociaux de sexe constituent-ils l'objet central ou périphérique de ces recherches? L'appartenance sexuelle est-elle traitée de concert avec d'autres types d'appartenance, comme la classe sociale, la race ou l'ethnie? Les cadres d'analyse féministe font-ils partie des approches théoriques? Que nous apprennent ces études? Peut-on formuler l'hypothèse du maintien ou de la progression des femmes aux études postsecondaires dans les pays industrialisés au cours des années à venir? 


\section{ABSTRACT}

\section{Social Interaction of the Sexes, Class and Race Affiliations, and Success in Higher Education: State of the Art Report}

Renée Cloutier, Faculty of Education Sciences, Université Laval, Canada

Since female students are in the majority in first cycle university studies in the United States, England and Canada, it may appear that gender affiliation is no longer considered a major issue in studies on success in higher education. Do studies on social sciences applied to education, published in scientific articles in 2002, deal with this phenomenon? Do researchers tend to pay more attention to other social groups because of the progress women have made? Is the social interaction of the sexes a central or peripheral subject in this research? Is gender affiliation treated in concert with other types of affiliations, such as social class, race or ethnic group? Are feminist analysis frameworks part of theoretical approaches? What do these studies teach us? Can we formulate the hypothesis that women's success in higher education will continue to progress in the years to come?

\section{RESUMEN}

\section{Las relaciones sociales de sexo, las membresías de clase y de raza y el éxito en enseñanza post-secundaria: balance de la cuestión}

Renée Cloutier, Facultad de ciencias de la educación, Universidad Laval, Canadá

El hecho que las mujeres sea mayoritarias en los estudios colegiales y en primer ciclo universitario en Estados Unidos, Inglaterra y Canadá puede hacernos pensar que la membresía sexual ya no se considera como uno de la retos más importantes en los estudios sobre el éxito educativo en la enseñanza post-secundaria ( $h i g h e r$ education). ¿Se constata este fenómeno en los estudios sobre el tema publicados en las revistas científicas en ciencias sociales aplicadas a la educación en 2002? ¿La progresión de las mujeres provoca que los investigadores e investigadoras les ofrezcan una menor atención en beneficio de otros grupos sociales? ¿Se aborda la pertenencia sexual junto con otros tipos de membresía, como la de clase social, de raza o de etnia? ¿Los cuadros de análisis feminista forman parte de esos enfoques teóricos? ¿Que nos enseñan esos estudios? ¿Podemos formular la hipótesis del mantenimiento o de la progresión de las mujeres en lo que se refiere al éxito educativo en enseñanza postsecundaria en los países industrializados en los próximos años? 


\section{1 - Le questionnement, les objectifs et la méthode1}

Depuis un grand nombre d'années, une des principales caractéristiques des études sociologiques en sciences de l'éducation a été le thème de la sélection et des inégalités sociales. Par exemple, l'origine socioéconomique des jeunes a été, au cours des 40 dernières années, dans les pays industrialisés, l'appartenance sociale qui a fait l'objet du plus grand nombre d'études dans le domaine de l'éducation, tant en France (Fave-Bonnet et Clerc, 2001; Marry, 2001) qu'aux États-Unis et en Angleterre (Dandurand et Ollivier, 1987). Puis, les appartenances raciale ou ethnique et l'appartenance sexuelle ${ }^{2}$ ont suivi comme enjeux et objets d'étude dans la foulée des revendications des mouvements féministes et des mouvements des droits civils. Il en a été de même au Québec, avec l'ajout des études sur l'appartenance linguistique (Dandurand et Ouellet, 1990).

Les femmes étant majoritaires aux études collégiales et au premier cycle universitaire tant aux États-Unis (Jacob, 2002; Karen, 2002), en Angleterre (David et al., 2003) qu'au Canada, on pourrait penser que l'appartenance sexuelle n'est plus considérée comme un des enjeux majeurs des études sur la réussite éducative en enseignement postsecondaire. Ce phénomène s'observe-t-il dans les articles sur ce thème publiés dans les revues scientifiques en sciences sociales appliquées à l'éducation en 2002? La progression des femmes fait-elle en sorte que les chercheurs et les chercheuses leur accordent moins d'attention au profit d'autres groupes sociaux? Les rapports sociaux de sexe constituent-ils l'objet central ou périphérique de ces recherches? L'appartenance sexuelle est-elle traitée de concert avec d'autres types d'appartenance, comme la classe sociale, l'ethnie ou la race? Les cadres d'analyse féministe font-ils partie des approches théoriques? Que nous apprennent ces études? Peut-on, en conclusion, après la discussion de ces résultats, formuler l'hypothèse du maintien ou de la progression des femmes en enseignement postsecondaire dans les pays industrialisés au cours des années à venir?

Ces questionnements découlent d'une première démarche d'analyse réalisée à partir de la recension d'écrits scientifiques, publiés en langues française ou anglaise en 2001 ou en $2000^{3}$ dans 48 revues scientifiques ${ }^{4}$ en sciences de l'éducation. J'ai mené cette première recherche avec trois étudiantes en sciences de l'orientation (Bellemare, 2003; Côté, 2003; Paré, 2003). Les articles choisis devaient porter sur l'un ou l'autre des thèmes ou dimensions suivantes : l'accès à l'éducation postsecondaire

1. Je remercie les évaluatrices ou évaluateurs anonymes, les membres du comité de rédaction ainsi que la rédactrice invitée du présent numéro, la professeure Jeanne d'Arc Gaudet de l'Université de Moncton, qui m'ont permis, grâce à leurs conseils judicieux, d'améliorer mon article. Je remercie de plus la vice-doyenne aux études supérieures et à la recherche à la Faculté des sciences de l'éducation de l'Université Laval, la professeure Geneviève Fournier, ainsi que le Centre de recherche et d'intervention sur l'éducation et la vie au travail (CRIEVAT) pour l'aide financière à la révision linguistique. Mes remerciements vont aussi à Hélène Dumais, linguiste, pour la révision linguistique du texte.

2. En France, d'après Catherine Marry, l'analyse, selon les inégalités de sexe par les sociologues, ne fait que de la « figuration » jusqu'aux années 80.

3. Les années 2001 ou 2000 ont été retenues lorsque les numéros de l'année 2001 n'étaient pas consultables sur les rayons de la bibliothèque à l'Université Laval en mars 2002.

4. Ce sont des revues avec comité de lecture. 
des étudiantes et des étudiants, leurs parcours ou cheminements durant les études et leur insertion socioprofessionnelle en fonction de la réussite éducative. Les études devaient dépasser la simple description pour inclure une analyse et une interprétation des données à partir des modèles analytiques des sciences sociales ${ }^{5}$ (sociologie, anthropologie, sciences économiques, sciences politiques, psychologie, sciences des organisations ${ }^{6}$ ). À partir de la table des matières des revues et du résumé des articles, nous avons sélectionné 46 articles publiés en 2001 ou en 2000 portant sur les thèmes retenus. Ces 46 articles ont constitué le corpus de la recension. L'objectif visé était la construction d'une typologie des principaux facteurs explicatifs de la réussite éducative qui se dégageaient des recherches (Cloutier et al., 2005a, 2005b) et la présentation systématique ${ }^{7}$ de chacun des écrits scientifiques à l'intérieur de cette typologie (Bellemare, 2003; Côté, 2003; Paré, 2003).

La recension des écrits de 2002 est en cours. Jusqu'à maintenant, j'ai dépouillé 29 revues scientifiques ${ }^{8}$ et $12^{9}$ d'entre elles ont publié 29 articles sur les mêmes thèmes ${ }^{10}$. Ces articles constituent le corpus de recherche pour la présente publication. Mon objet d'analyse porte cette fois sur les groupes sociaux ou les appartenances sociales comme facteurs explicatifs de la réussite éducative, avec pour préoccupation centrale les rapports sociaux de sexe. Dans l'analyse des articles publiés en 2001, j'avais observé que 28 des 46 études retenaient dans leur schéma d'analyse diverses appartenances sociales comme variables indépendantes dans l'explication de la réussite éducative en enseignement postsecondaire. Cet intérêt, pour ces appartenances et l'éclairage auquel elles pouvaient contribuer, ne m'a pas semblé " anormal », mais au contraire "normal», dans la lignée des préoccupations des chercheuses et des chercheurs en sciences sociales appliquées à l'éducation par rapport à la question des inégalités sociales.

Cette observation a stimulé mon désir de poursuivre l'analyse dans ce sens et de montrer la contribution des études en sciences sociales quant à la compréhension de la réussite éducative en enseignement postsecondaire, principalement aux ÉtatsUnis, en Angleterre et au Canada ${ }^{11}$. En 2002, outre les études de ces pays ${ }^{12}$, d'autres

5. Il était parfois difficile, à partir de la note biographique des auteures et des auteurs, de connaître leur appartenance disciplinaire. Dans ces cas, la sélection des articles a eu lieu à partir des modèles théoriques, conceptuels et empiriques.

6. Pour la recension en 2002, j'ai examiné en outre des revues en histoire de l'éducation.

7. Les rubriques suivantes ont guidé le dépouillement de chacun des articles: I'appartenance disciplinaire et institutionnelle des auteures et des auteurs, leurs buts ou les objectifs visés dans l'article, le contexte (social ou politique), les références théoriques, conceptuelles et empiriques, le modèle théorique et conceptuel adopté par les auteures et les auteurs, les hypothèses de recherche, l'échantillon, le mode de collecte des données, la catégorisation des principales variables (dépendantes, indépendantes et témoins), le traitement des données, les principaux résultats de l'étude, les pistes de recherche ainsi que les pistes d'intervention proposées.

8. Voir l'annexe I.

9. Certaines de ces revues ont publié d'autres articles en 2002 sur les thèmes répertoriés. Ces articles seront traités dans une publication ultérieure.

10. Voir l'annexe II.

11. En 2001 ou en 2000, les données des études proviennent des États-Unis (18), de la France (10), du Canada (9), du Royaume-Uni (7), de la Suisse (1) et de divers pays (données de I'UNESCO et des Nations Unies) (1).

12. En 2002, les données des études proviennent des États-Unis (14), du Canada (6), du Royaume-Uni (4), de la France (1) et d'autres pays (5). 
études, parmi les 29 articles recensés, ont été menées en Israël ${ }^{13}$, en Chine ${ }^{14}$ (Hong Kong), aux Indes ${ }^{15}$ et en Australie ${ }^{16}$. Mentionnons toutefois que seulement 3 articles $^{17}$ incluent dans leur échantillon des étudiantes et des étudiants des deuxième et troisième cycles (maîtrise ou doctorat), la majorité des études portant plutôt sur les ordres d'enseignement (collégial ou premier cycle universitaire) ou sur les établissements qui les donnent. La même tendance avait été observée en 2001.

\section{1 - La catégorisation des études selon les appartenances sociales et la préséance accordée aux rapports sociaux de sexe}

J'ai donc amorcé la recension des écrits de 2002 en catégorisant d'abord les études selon le nombre d'appartenance sociale faisant l'objet de l'analyse et de la diversité de ces dernières. Dans un premier temps, j'ai classé les articles par groupe selon qu'ils accordaient préséance aux rapports sociaux de sexe et aux femmes et qu'ils privilégiaient une approche sociologique féministe dans leurs analyses ou encore qu'ils s'en éloignaient ou n'en tenaient pas compte. Les critères suivants m'ont guidée dans ce classement:

- la mention, dans le titre de l'article, du ou des termes suivants : femmes, genre (women ${ }^{18}$, gender $\left.{ }^{19}\right)$, sexe $\left(\operatorname{sex}^{20}\right)$, différence de genre ou de sexe (gender differences $^{21}$, sex differences ${ }^{22}$ ), écart entre les sexes (gender gap ${ }^{23}$ ), masculinité (masculinity ${ }^{24}$ ), garçons (boys ${ }^{25}$ ). Au total, 10 articles sur 29 nommaient dans le titre l'un ou l'autre de ces mots;

- la mention, dans la description des caractéristiques de l'échantillon, des femmes ${ }^{26}$ ou des hommes et des femmes encore aux études ou anciens étudiants et étudiantes ${ }^{27}: 4$ articles $^{28}$ ne précisaient pas le sexe;

- les femmes ${ }^{29}$ ou les hommes et les femmes toujours aux études ou anciens étudiants et étudiantes comme un des critères de sélection ${ }^{30}$ de l'échantillon;

- l'appartenance sexuelle comme faisant partie des variables principales indépendantes ${ }^{31}$ ou témoins ${ }^{32}$, ou des variables indépendantes et témoins ${ }^{33}$;

13. Voir l'article 3 de l'annexe II.

14. Voir l'article 22 de l'annexe II.

15. Voir l'article 8 de l'annexe II.

16. Voir l'article 1 de l'annexe II.

17. Voir les articles 1,20 et 22 de l'annexe II.

18. Voir les articles 3,9 et 11 de l'annexe II.

19. Voir l'article 8 de l'annexe II.

20. Voir l'article 4 de l'annexe II.

21. Voir l'article 6 de l'annexe II.

22. Voir l'article 20 de l'annexe II.

23. Voir l'article 14 de l'annexe II.

24. Voir l'article 7 de l'annexe II.

25. Voir l'article 14 de l'annexe II.

26. Voir les articles 1,3 et 14 de l'annexe II qui avaient comme échantillon des étudiantes.

27. On compte 22 articles dans cette catégorie.

28. Voir les articles 26, 27, 28 et 29 de l'annexe II.

29. Voir les articles 1,3 et 9 de l'annexe II.

30. Voir les articles $1,2,3,5,6,7,8,11,14,20,21$ et 22 de l'annexe II.

31. Voir les articles $1,2,4,9,14$ et 20 de l'annexe II.

32. Voir l'article 6 de l'annexe II.

33. Voir les articles 8 et 21 de l'annexe II. 
- le fait que l'un des paradigmes théoriques ou conceptuels privilégiés par les chercheuses et les chercheurs s'inscrit dans le courant des inégalités sociales liées aux rapports sociaux de $\operatorname{sex}^{34}$;

- le fait que l'un des cadres d'analyse est féministe ${ }^{35}$;

- la mention dans les références des articles du ou des termes suivants : féminisme ou féministe (feminist), discrimination sexuelle (sex discrimination), égalité des sexes (sex equality), femmes, genre (women, gender), sexe (sex), différence de genre ou de sexe (gender differences, sex differences), écart entre les sexes (gender gap), hommes/femmes (men/women, male/female), filles (girls), garçons (boys), fils (son), etc.

\section{2 - Les résultats}

\section{1 - Les diverses appartenances sociales}

Tout comme je l'avais observé dans les écrits scientifiques de 2001, le sexe est le facteur privilégié (tableau 1) parmi les types d'appartenances sociales faisant l'objet de variables indépendantes ou témoins dans les schémas explicatifs des articles de 2002. L'appartenance sexuelle n'est donc pas évacuée des études sur la réussite éducative en enseignement supérieur.

\section{Tableau 1 : Les principales appartenances sociales (2002)}

\begin{tabular}{|l|l|}
\hline Sexe & 25 \\
\hline Origine socioéconomique & 18 \\
\hline Race ou ethnie & 16 \\
\hline Résidence (milieu rural ou urbain, province ou État, région géographique, immigration) & 07 \\
\hline Âge & 04 \\
\hline Total (articles) & 29 \\
\hline
\end{tabular}

L'appartenance sexuelle est rarement étudiée seule en 2002 (tableau 2), comme c'était le cas dans les études de 2001 (Cloutier et al., 2005a). Elle est examinée de façon concomitante avec d'autres types d'appartenances, les principales étant la race ou l'ethnie et l'origine socioéconomique.

34. Voir les articles $1,2,3,4,5,6,7,8,9,10$ et 11 de l'annexe II.

35. Voir les articles $1,2,3,4,5$ et 10 de l'annexe II. 
Tableau 2 : Des groupes sociaux ou des appartenances sociales : variables indépendantes ou témoins de la réussite éducative (2002)

\begin{tabular}{|c|c|}
\hline Une appartenance sociale & 06 \\
\hline Sexe (articles $1,4,22$ et $20 *$ ) & (4) \\
\hline Race ou ethnie (articles 27 et 29) & (2) \\
\hline Deux appartenances sociales & 08 \\
\hline Sexe et origine socioéconomique (articles 7,2 , et 19 ) & (3) \\
\hline Sexe et race ou ethnie (articles 24 et 11 ) & (2) \\
\hline Sexe et ethnie nationale (article 3 ) & (1) \\
\hline Origine socioéconomique et race (article 26) & (1) \\
\hline Origine socioéconomique et résidence (milieu rural ou urbain) (article 28) & (1) \\
\hline Trois appartenances sociales & 06 \\
\hline Sexe, race ou ethnie et origine socioéconomique (articles 5,13 et 12) & (3) \\
\hline Sexe, statut d'immigration et région géographique d'immigration (article 16) & (1) \\
\hline Sexe, origine socioéconomique et âge (article 25) & (1) \\
\hline Sexe, âge et résidence (milieu rural ou urbain) (article 8) & (1) \\
\hline Quatre appartenances sociales & 06 \\
\hline $\begin{array}{l}\text { Sexe, race ou ethnie, origine socioéconomique et résidence } \\
\text { (milieu rural ou urbain) (articles } 6,15 \text { et 18) }\end{array}$ & (3) \\
\hline Sexe, race ou ethnie, origine socioéconomique et âge (articles 23 et 9) & (2) \\
\hline Sexe, origine socioéconomique, âge et résidence (province ou État) (article 21) & (1) \\
\hline Quatre appartenances sociales et plus, dont celle du sexe (articles 14,17 et 10 ) & 03 \\
\hline Total (articles) & 29 \\
\hline
\end{tabular}

" Les chiffres font référence aux articles répertoriés dans l'annexe II.

\section{2 - Les rapports sociaux de sexe et les approches féministes}

Dans un second temps de la catégorisation de ces données, j'ai classé les articles en dix groupes selon qu'ils accordaient plus ou moins préséance aux rapports sociaux de sexe et aux femmes et qu'ils privilégiaient ou non une approche sociologique féministe dans leurs analyses. Je me suis inspirée en outre pour ce classement ${ }^{36}$ des travaux suivants : Descarries-Bélanger et Roy (1988); Juteau et Laurin (1988); DauneRichard et Devreux, (1992); Mosconi (1995); Bouchard, Cloutier et Hamel (1996); Collectif Laure-Gaudreault (1997); Descarries (1998); David et al. (2003). Le classement final répartit les articles sur un continuum, le groupe 1 réunissant les articles féministes et le groupe 10, ceux qui n’ont pas retenu le sexe parmi les appartenances sociales (voir l'annexe II pour le titre des articles) :

$\sum \cdot$ Groupe 1 : Le cadre d'analyse est féministe (articles 1-4);

$\Sigma \bullet$ Groupe 2: Un des cadres d'analyse est féministe (article 5);

36. Cela a été le cas en particulier pour la classification des articles selon une approche sociologique féministe. 
$\Sigma \cdot$ Groupe 3: Un des cadres d'analyse s'inscrit dans le courant des inégalités sociales liées aux rapports sociaux de sexe (articles 6-9);

$\Sigma$ - Groupe 4: Un des cadres d'analyse est féministe et l'analyse porte principalement sur l'appartenance raciale ou ethnique (article 10);

$\Sigma \cdot$ Groupe 5: Un des cadres d'analyse s'inscrit dans le courant des inégalités sociales liées aux rapports sociaux de sexe et l'analyse porte principalement sur l'appartenance raciale ou ethnique (article 11);

$\Sigma$ - Groupe 6: Un des cadres d'analyse s'inscrit dans le courant des inégalités sociales liées aux appartenances sociales, dont l'appartenance sexuelle (articles 12-20);

$\Sigma \cdot$ Groupe 7: Le sexe est considéré comme une variable indépendante et comme une variable témoin (article 21);

$\Sigma \cdot G r o u p e$ 8: Le sexe est considéré comme une des variables témoins (article 22);

$\Sigma \cdot$ Groupe 9: Le sexe est considéré comme une des variables témoins, sans analyse particulière (articles 23-25);

$\Sigma \cdot$ Groupe 10: Le sexe n'a pas été retenu comme variable parmi les appartenances sociales (articles 26-29).

À noter que, dans la section 2.2.1, je traite des 11 articles qui situaient davantage leurs analyses dans le champ des études féministes (groupes 1 à 5). Néanmoins, j’ai utilisé les résultats des recherches des autres auteures et auteurs (groupes 6 à 10) dans l'analyse et la discussion des résultats (voir la section 3).

Chez les féministes, le concept de «travail» inclut à la fois le travail invisible, non rémunéré et non reconnu.

\subsection{1 - Les recherches dans le champ des études féministes ${ }^{37}$}

Que nous apprennent ces études sur les thèmes de l'accès à l'enseignement postsecondaire des étudiantes et des étudiants, de leurs parcours ou cheminements durant les études et de leur insertion socioprofessionnelle en fonction de la réussite éducative $^{38}$ ? J'ai regroupé les trois premières sous le thème du travail tel qu'il a été défini et élaboré par des sociologues féministes (Delphy, 1970, 1998). Chez les féministes, le concept de « travail » inclut à la fois le travail invisible, non rémunéré et non reconnu qui est fourni par les femmes dans la sphère familiale (mise au monde, soin et éducation des enfants ainsi que tâches domestiques) et le travail visible, salarié en emploi. On verra plus loin que la conciliation du travail invisible et du travail visible pose encore problème aux femmes.

37. Afin de respecter le langage employé dans chacun des articles répertoriés, j'ai féminisé le texte dans cette section de mon article dans les cas où les chercheuses et les chercheurs le faisaient ou encore dans les cas où j'avais des informations précises par rapport au sexe des personnes ayant fait l'objet des études. Pour les autres parties de mon article, j'ai féminisé le texte selon les normes de féminisation adoptées par la revue Recherches féministes.

38. Cette section est plus longue que les normes habituelles pour un article publié dans une revue scientifique. Il m'est apparu difficile, compte tenu du corpus de recherche, de faire plus bref. Je vise aussi, à travers cette section, à initier des étudiantes et des étudiants universitaires aux recherches en sciences sociales appliquées à l'éducation. 


\subsubsection{1 - Le travail invisible et le travail visible des femmes}

Le premier article considéré est une étude longitudinale qualitative menée en Australie de 1986 à 2001 auprès de jeunes femmes performantes sur le plan scolaire. Celles-ci venaient de divers milieux sociaux, avec prédominance de la classe moyenne. Cette étude permet d'examiner la réussite éducative sous l'angle de l'insertion professionnelle de ces jeunes femmes et de leurs possibilités d'atteindre l'ensemble de leurs objectifs (mariage, enfants et carrière) tels qu'elles les avaient exprimés ${ }^{39}$ en 1986 au moment où elles fréquentaient l'école secondaire ou l'université à Perth, en Australie de l'Ouest. Ces jeunes femmes avaient été éduquées en Australie au moment où les discours et les pratiques sur l'«égalité entre les sexes » en éducation florissaient.

Nado Aveling (2002) rappelle en introduction de son étude ${ }^{40}$ certains des objectifs du projet féministe qui sont de libérer les femmes "de pratiques et de structures " qui ont perpétué la position inégale des femmes dans la sociétét1. Compte tenu des changements sociaux importants qui se sont produits depuis les années 60 dans les sociétés postindustrielles, les choix de vie (lifestyle choices ${ }^{42}$ ) qui s'offrent aux femmes sont plus diversifiés et plus complexes que ceux qui étaient accessibles à leurs mères une génération plus tôt. À cette période, poursuit Aveling, la question qui préoccupait les chercheurs était de savoir pourquoi certaines femmes « choisissaient de travailler » et d'autres non. Cette question n'est plus pertinente aujourd'hui dans des pays comme l'Australie, ni dans d'autres nations industrialisées de l'Ouest, où les femmes disent qu'elles s'attendent (expecting) à la fois de se marier, d'avoir des enfants et de faire carrière. Par ailleurs, d'autres études ont montré, rapporte Aveling, que les femmes sont encore les principales responsables des soins aux enfants. Qu'en sera-t-il alors de la réalisation de leurs aspirations de travail (famille et emploi)?

L'échantillon initial d'Aveling est composé d'un groupe de 63 femmes $^{43}$. La chercheuse voulait, dans cette première collecte de données, examiner avec ces femmes les avenues qu'elles avaient explorées afin de prendre leurs décisions par rapport à leur future carrière et les chemins qu'elles envisageaient de suivre au cours de leur vie (life-paths). Aveling est demeurée en contact avec ces jeunes femmes au cours des années subséquentes pour vérifier comment les désirs exprimés en 1986 se conjuguaient avec la réalité des femmes vivant et travaillant en Australie au tournant du millénaire. En 2000-2001, la chercheuse a mené des entrevues avec 12 de ces femmes. C'est sur ces dernières données que l'auteure a porté principalement son attention dans son article, avec comme point comparatif pour son analyse le discours

39. "To have it all!», selon leurs expressions.

40. Nado AVELING (2002), " "Having It All" and the Discourse of Equal Opportunity: Reflections on Choices and Changing Perceptions ", Gender and Education, 14, 3: 265-280 (article 1).

41. Ways of changing women's position in society for the better-to liberate women from practices and structures which have perpetuated women's unequal position in society-have, by definition, always been part of the feminist project » (Aveling, 2002: 265).

42. Je laisse volontairement des expressions en anglais tout au long du texte afin de respecter le langage conceptuel des différentes équipes de recherche.

43. L'échantillon initial est composé de 63 femmes. La moyenne d'âge du sous-échantillon des plus jeunes femmes (17) est de 17 ans en 1986; celle des plus vieilles (46), de 23 ans. 
de l'«égalité entre les sexes» à l'origine des réformes en éducation en Australie durant les années $80^{44}$, période où les filles de l'échantillon fréquentaient l'école.

En 1986, ces femmes expriment l'idée que leur processus de décision n'a pas été marqué par leur sexe ni par leurs projections futures de se marier et d'avoir ou non des enfants. Leur discours par rapport à leur éducation aussi bien que celui par rapport au marché du travail a comme cadre d'analyse celui de l'égalité entre les sexes, même si leurs familles semblent leur avoir montré l'existence de positions inégalitaires à la faveur des hommes dans la société. Les portes du marché du travail leur sont ouvertes, et ces femmes espèrent que l'ensemble de leurs désirs vont se concrétiser ${ }^{45}$.

En 1998, parmi les 37 femmes qui ont répondu au questionnaire, 2 avaient obtenu un diplôme d'un collège technique, 22 avaient obtenu un baccalauréat en arts ou en sciences et 6 étaient titulaires d'un diplôme supérieur. Pour ce qui est de l'emploi, la majorité des femmes qui avaient des projets particuliers (médecine, droit, recherche scientifique, enseignement) les ont réalisés. Celles qui n'avaient pas de projet précis en fait d'occupation disent avoir trouvé un travail salarié dans un domaine dans lequel elles avaient toujours eu un intérêt. Plusieurs mentionnent aimer leur travail et faire bon usage de leur qualification professionnelle. Il n'y a pas de différence dans les commentaires des femmes selon leur âge. La principale différence dans la transformation de leur réalité est plutôt liée à la maternité. Toutes les mères (8) considèrent que c'est leur plus importante réalisation (achievement) jusqu'à ce jour, bien qu'elles aient dû réduire leurs activités professionnelles, exception faite de celle qui est chef de famille et qui occupe un emploi à plein temps. Leurs discours, en particulier pour celles qui sont dépendantes financièrement du salaire de leur conjoint, se modifient toutefois par rapport à celui de 1986: «ces femmes semblent avoir le sentiment qu'obtenir le «meilleur» de l'ensemble des possibilités qu'elles avaient envisagées n'est pas la même chose que de réaliser «l'ensemble » de leurs aspirations ${ }^{46}$ ». Et toutes les mères reconnaissent que leur réalité n'est pas ce qu'elles avaient imaginé.

En 2000-2001 ${ }^{47}$, soit quinze ans après la première entrevue, le discours de ces femmes éclaire encore davantage les changements de perception de leurs réalités ${ }^{48}$. La démarcation est plus prononcée qu'en 1998 entre celles qui ont des enfants (6) et celles qui n'en ont pas. Leurs modèles d'emploi reflètent ceux qui ont émergé dans les questionnaires de 1998. Ils illustrent en outre leur statut maternel ${ }^{49}$ plutôt que leur scolarité et leur désir d'occuper un emploi. Les mères se consacrent à temps plein à leur travail auprès de leurs enfants ou occupent conjointement un emploi à

44. "Labor governments were formally committed to programmes of gender reform through a system of tied grants to the states (who have responsability for education) » (Aveling, 2002: 268).

45. "I hope my dreams come true to "have it all"! » : tel était le résumé de leurs discours en 1986.

46. "[Those] women [...] seemed to have a sense that " having the best of all possible worlds " was not quite the same thing as their desires to " have it all" » (Aveling, 2002: 273).

47. Parmi les 12 femmes rencontrées en entrevues, 5 faisaient partie du sous-échantillon " jeunes » en 1986 et 7, de celui des plus âgées.

48. " [...] the ways in which these women's desire to " have it all " worked itself out in practice " (Aveling, 2002: 274)

49. "They reflected not their education or their commitment to work, but their maternal status » (Aveling, 2002 : 274). 
temps partiel. Cette tendance observée ici est la même que pour la population féminine en général en Australie en 1999-2000 ${ }^{50}$. Les femmes, lorsqu'elles sont mères, changent en effet leurs pratiques pour assumer la responsabilité quotidienne parentale et modifient également leurs discours pour donner une signification à leur vie. « Ma vie a changé! », tel est le résumé de leurs propos:

Je me suis souvent demandée : «Qu'est-ce j'avais fait? Qu'est-ce que j'avais accompli? » J'ai eu deux merveilleux enfants; mon mari aussi, mais il a obtenu en plus un doctorat et un merveilleux emploi (Eve) ${ }^{51}$.

Quand mon partenaire et moi avons discuté de qui va s'occuper des enfants et que j'ai dit: «Bien, mon salaire est plus élevé que le tien», il a répondu : ¿C’est vrai, mais si je ne travaille pas, je mets mes compétences en veilleuse, et alors ce sera beaucoup plus difficile pour moi lorsque je retournerai dans ce secteur ", et, à la fin de nos discussions, c'est lui qui l'a emporté [...] Je ne conçois pas que c'est sa faute ou quelque chose de fait de façon délibérée, mais le fait que la société attende de lui qu'il aille de l'avant et travaille à temps plein, et je pense que si j'avais un conjoint différent, si j'avais le type de conjoint que je considère comme idéal, dans le sens qu'il soit capable d'assumer ses responsabilités parentales, cela aurait été un contexte complètement différent-une réalité complètement différente-mais où pouvez-vous trouver des hommes comme cela? (Ingrid) ${ }^{52}$.

Aveling conclut son article en ces termes. Le discours sur l'égalité des chances pour les femmes et les hommes a erré ( $h a d$ failed) sur un certain nombre d'aspects. Les filles ont certainement amélioré leurs positions, comme groupe, par rapport aux garçons pour ce qui est de leur participation scolaire et de ses retombées. Cependant, pour celles qui sont mères, leurs modèles de travail reproduisent ceux des femmes de la génération antérieure ${ }^{53}$.

Afin que les femmes puissent avoir les mêmes types d'occasions que les hommes sur le marché du travail, il ne suffit pas qu'elles soient traitées de la même façon que les garçons à l'école. Ce traitement scolaire égal ne garantit pas non plus une participation égalitaire selon le sexe dans la sphère domestique (Aveling, 2002: 278). Les

50. Une baisse importante de l'emploi à temps plein et à temps partiel s'observe, et de façon plus marquée pour les femmes âgées de 25 à 34 ans, et elle est encore plus accentuée pour celles dont l'âge varie de 35 à 44 ans: Australian Bureau of Statistics (2001) cité dans Aveling (2002 : 274).

51. "I often asked myself what have I done? What have I achieved? I mean I've got two wonderful children, but so has my husband and he's got a PhD and a wonderful job (Eve) » (Aveling, 2002: 276).

52. " [When] my partner and I had the discussion about who was going to be it (s'occuper de leur enfant), and I said, well, I'm earning more than you are, and he said, well, but if I don't work, I lose my skills and then it's much for me to back into my field, and in the end that's what did it [...] I don't see it as his fault, or something that he's deliberately done, but the fact is that society expects him to go off and work full-time, and I think probably if I had a different partner, if I had the sort of partner that I would consider as being ideal, in that sense of being able to take responsability for parenting, so that would have been a completely different set of circomstances-a completely different outcome-but you know where do you find men like that? (Ingrid) » (Aveling, 2002: 279).

53. "Certainly, girls as a group have markedly improved their positions about boys in terms of educational outcomes and participation (L. Yates, 1993b; Gender Equity Taskforce, 1997; L. McLeod, 1998) [...] their work patterns essentially replicated the employment patterns of women of an earlier generation » (Aveling, 2002: 277). 
Des changements doivent être apportés aux programmes d'études afin que les jeunes hommes développent les diverses habiletés liées à la paternité. enseignantes et les enseignants doivent être conscients que les programmes d'études désavantagent les filles, notamment par le silence au sujet des femmes comme mères et des hommes comme pères. Des changements doivent être apportés aux programmes d'études afin que les jeunes hommes développent les diverses habiletés liées à la paternité. Des études doivent donc être réalisées dans ce champ.

La situation de ces jeunes femmes australiennes est-elle si différente de celle de femmes et d'hommes diplômés au cours des années 30 d'universités anglaises et de collèges universitaires? La deuxième étude (article) ${ }^{54}$ examinée ici a été menée en Angleterre par Carol Dyhouse (2002). Celle-ci avait pour objectifs d'explorer trois questions ou enjeux : 1) Pourquoi la génération de femmes qui a obtenu son diplôme juste avant la Seconde Guerre mondiale, à une époque où ce n'était pas un comportement routinier pour les femmes, a-t-elle choisi d'aller à l'université et qu'espérait-elle (et leurs familles) en retour? 2) Quels rôles leurs parents, et spécialement leurs mères, ont-ils joué en les envoyant aux études et en les soutenant dans la poursuite de leurs études universitaires? 3) Comment des femmes qui ont obtenu leurs diplômes pendant les années 30 considèrent-elles (durant les années 90) qu'elles ont «bénéficié » de leurs études universitaires, et comment évaluent-elles les retombées de l'enseignement supérieur en relation avec l'expérience des femmes?

Selon l'auteure, la forte progression de la demande d'admission aux études supérieures à travers les générations en Angleterre peut être mieux circonscrite par la connaissance de la scolarisation des femmes au milieu du XXe siècle. Dyhouse souhaite contribuer aux études historiques ayant pour objet de redonner de la visibilité aux femmes et à leurs influences dans les modèles de mobilité sociale au siècle dernier. La chercheuse essaie dans son étude d'explorer la signification sociale de l'expansion de l'enseignement supérieur durant la première moitié du XXe siècle à partir de son étude auprès de diplômées et de diplômés des années 30 au cours des années 90. Les établissements d'enseignement supérieur ont été choisis de façon à refléter le portrait des institutions ${ }^{55}$, à l'exception des universités d'Oxford et de Cambridge. Plus de 500 femmes et 600 hommes ont répondu au questionnaire de Dyhouse. Celui-ci fournit des données sur les antécédents sociaux des titulaires d'un diplôme, leur expérience éducationnelle et leurs histoires familiale et professionnelle. Dans ce questionnaire, Dyhouse a aussi demandé aux personnes interrogées d'exprimer les motifs de leur poursuite d'études universitaires et la façon dont elles les avaient financées. La chercheuse utilise également comme matériel pour sa démonstration des ouvrages scientifiques sur la scolarisation des femmes et des hommes, principalement pendant la première moitié du XXe siècle, et son effet sur la mobilité sociale. Dyhouse inscrit sa recherche dans le cadre d'analyse qui reconnaît que les retombées d'un diplôme en enseignement supérieur, en particulier pour les femmes de cette époque, ne peuvent être étudiées uniquement sous l'angle du ren-

54. DYHOUSE, Carol (2002), " Graduates, Mothers and Graduate Mothers: Family Investment in Higher Education in Twentieth-Century England », Gender and Education, 14, 7: 325-336 (article 2).

55. Cela comprend les plus récentes et les plus anciennes institutions, des universités dans diverses parties du pays ainsi que des établissements mixtes et séparés (Dyhouse, 2002 : 327). 
dement ou du capital financier ${ }^{56}$. L'analyse doit en outre être faite sous l'angle du capital culturel et de la mobilité sociale pour ces femmes et leur progéniture, de même que par rapport à une meilleure qualité de vie. Elle confirmera d'ailleurs cette tendance dans son étude, comme on le verra plus loin.

Les principaux résultats de l'étude de Dyhouse concernant ses trois questions ou enjeux sont les suivants. Parmi les raisons qui ont incité ces personnes à poursuivre des études universitaires, $67,8 \%$ des hommes et $37,5 \%$ des femmes mentionnent des objectifs professionnels clairs, ces pourcentages grimpant à $81,3 \%$ et à $51,2 \%$ avec l'ajout des objectifs professionnels "généraux" (Dyhouse, 2002: 328). Ces résultats soulèvent la question à savoir si ces femmes sont des "consommatrices » de l'éducation, recherchant la connaissance et la culture en soi, par opposition à une visée de l'éducation qui constituerait un passeport pour gagner sa vie ${ }^{57}$. D'autres réponses et commentaires fournis par les diplômées révèlent que la grande majorité d'entre elles indiquent des raisons économiques: assurer (to earn) leur propre subsistance, aider financièrement leurs parents ou apporter leur soutien à la scolarisation de leurs frères et sœurs (siblings).

Au total, 54,5\% des femmes de l'échantillon et 43,3\% des hommes viennent de la classe moyenne, et un peu moins de femmes viennent de la classe de la maind'œuvre semi-qualifiée. Par ailleurs, $39 \%$ des hommes et $41 \%$ des femmes mentionnent que leurs familles ont payé « tout ou presque tout » du coût de leurs études universitaires ${ }^{58}$. Cependant, cette contribution financière ne provient pas uniquement de leurs parents. D'autres membres de la famille (grands-parents, frères et sœurs (siblings), etc.) y ont aussi contribué. Une plus grande proportion de femmes $(82,2 \%)$ que d'hommes $(46,3 \%)$ précisent avoir reçu un soutien important de leurs parents dans la poursuite de leurs études universitaires, les mères, encore plus que les pères, offrant à leurs filles et à leurs fils un tel encouragement. Toutefois, seulement dix femmes soulignent que leurs pères ont été réticents à ce qu'elles poursuivent leurs études. Dans cette situation, ces femmes ont indiqué que leurs mères étaient intervenues en leur faveur. Les mères les plus scolarisées ont aidé leurs filles sur le plan scolaire en leur transmettant leurs propres connaissances. Les mères qui n'avaient qu'une faible scolarisation ont souvent offert leurs services ${ }^{59}$ afin que leurs filles ou leurs fils poursuivent leurs études. Dyhouse confirme ainsi dans son étude des résultats de recherche sur la mobilité sociale, selon lesquels les mères ont une plus forte influence sur leurs filles en matière d'ambitions éducationnelles et de carrière, les pères influençant davantage leurs fils. Cette influence confirmerait les explications fournies par les théories de l'apprentissage des rôles sexuels ou de la socialisation ${ }^{60}$ en particulier dans les familles de classe moyenne (Heward, 1988; Dyhouse, 2001

56. Je reviendrai sur ce thème à la section suivante: 2.2 .1 .2

57. Howarth et Curthoys (1987) cités dans Dyhouse (2002: 328).

58. Doreen Whitely (1933) a estimé que plus de la moitié des étudiantes et des étudiants de premier cycle bénéficiaient d'une forme ou l'autre de bourse (from some kind of sholarship or bursary) dans les années 30. Dyhouse rappelle en outre que près de la moitié de ces étudiants, autres que ceux d'0xford et Cambridge, fréquentaient une université locale et habitaient chez leurs parents (Dyhouse, 2002 : 328-329).

59. Cela consistera à prendre des pensionnaires à la maison, à s'occuper de la lessive d'autres familles, à faire le ménage dans des foyers, etc.

60. Miller et Hayes (1990: 63) cités dans Dyhouse,(2002: 330). 
cités dans Dyhouse, 2002 : 330). Dyhouse constate cependant, pour les fils issus des classes sociales moins élevées, que la mère influence davantage ses fils que le père dans la médiation entre deux formes de masculinité liée au travail ${ }^{61}$.

Si la presque totalité des hommes de l'échantillon a connu une mobilité sociale (carrière), la situation est toutefois moins claire du côté des femmes. Et il est difficile encore ici, selon la chercheuse, de résumer les informations au sujet du travail rémunéré des femmes. Un peu plus de la moitié (51\%) des femmes diplômées ont occupé un emploi de façon plus ou moins continue jusqu'à leur retraite ${ }^{62}$. Un peu plus du tiers des femmes ont consacré une partie importante de leur temps à élever leurs enfants et sont retournées par la suite sur le marché du travail rémunéré à temps plein ou à temps partiel. Moins de $1 \%$ des femmes ont mentionné qu'elles n'étaient jamais entrées sur le marché de l'emploi au cours de leur vie (Dyhouse, 2002 : 332). En général, poursuit Dyhouse, les réponses de ces femmes témoignent du statut contradictoire et controversé de la participation des épouses et des mères au marché de l'emploi durant les années 50 et 60 en Angleterre ${ }^{63}$.

La très grande majorité des femmes interrogées n'expriment toutefois aucun doute sur la "valeur» de leur éducation universitaire ${ }^{64}$. Les hommes traduisent moins leur pensée sous ce registre. Néanmoins, ils considèrent que «leur formation en enseignement supérieur a été " essentielle » ou que leur diplôme a été très important dans la recherche d'un premier contrat de travail 65 ». Les hommes ont toutefois mentionné que leur expérience, dans le Service national lors de la Seconde Guerre mondiale, a été aussi importante que leur éducation universitaire concernant le cheminement de leur carrière..

En outre, la chercheuse a pu constater les retombées, pour ces femmes et ces hommes, de leur poursuite d'études postsecondaires en fait de capital culturel "transmis" ou légué. Plusieurs ont exprimé, même si aucune question ne portait directement sur ce sujet, leur fierté quant au fait que bon nombre de leurs enfants et petits-enfants ont mené des études universitaires.

La troisième recherche examinée a comme échantillon des femmes américaines âgées de 15 à 44 ans, de diverses appartenances sociales (socioéconomique ${ }^{66}$ et

61. "Many of these mothers might be seen as having played an important part in mediating between two class-based forms of masculinity between the wars. As more traditional, work-based forms of maculinity tied with apprenticeship, skill and regular wages were increasingly undermined by depression, the prospect of staying on in education began to look like a wise investment » (Dyhouse, 2002: 331).

62. Ce pourcentage regroupe en outre les travailleuses célibataires (33\%), la plupart des femmes mariées qui n'ont pas eu d'enfants ainsi que les femmes médecins et les enseignantes (Dyhouse, 2002: 332).

63. "In the main, graduate status conferred far less clear-cut social and economic advantages on women than on men; and for this generation of graduate wives and mothers, the question of their participation in paid employment was complex and controversial. My questions about work evoked detailed answers, but many of the replies were troubled, combative or defensive in tone. It is clear that even at this late stage in their lives, there were issues that were unresolved, and that women were haunted by the legacy of those controversies of the 1950s and 1960s » (Dyhouse, 2002: 332).

64. " [...] "it had made all the difference in the world" [...] "it was a gift beyond price", "a sort of heaven on earth" [...] Personal enrichment and the quality of lifelong friendships were often mentioned » (Dyhouse, 2002: 333-334).

65. "[Their] higher education was "essential", or that their degrees had been important in helping them secure a first appointment 》 (Dyhouse, 2002 : 334).

66. Le statut socioéconomique est mesuré par les niveaux de scolarité de la mère et du père. 
raciale ou ethnique ${ }^{67}$ ). L'objectif principal de l'étude menée par Jerry A. Jacobs et Rosalind Bertowitz King (2002) ${ }^{68}$ est de vérifier si la probabilité de terminer des études collégiales pour ces étudiantes varie avec l'âge. Cette problématique touche un grand nombre de personnes. En 1995, les étudiantes et les étudiants adultes inscrits au collège représentent un groupe important aux États-Unis. Plus du tiers (37,3\%) des étudiantes et des étudiants non diplômés sont âgés de 25 ans et plus :18,8 \% sont inscrits à temps plein, tandis que la majorité $(63,3 \%)$ le sont à temps partiel (U.S. Department of Education (1998) cité dans Jacobs et Bertowitz King (2002: 211)).

Les principales variables indépendantes ou facteurs explicatifs de cette étude sont les effets du temps, soit les diverses périodes d'inscription au collège des étudiantes et les reports de l'inscription ${ }^{69}$, ainsi que les effets des événements de vie (life events), tels que la naissance d'enfants, le mariage, le divorce et l'emploi, sur les probabilités pour les femmes de terminer des études collégiales. Peu de recherches sur ce sujet, écrivent Jacobs et Bertowitz King, retiennent à la fois les facteurs de l'origine socioéconomique des étudiants et des étudiantes et de leur histoire de vie (life events). Pour leur part, Jacobs et Bertowitz King utilisent les données de l'enquête américaine National Survey of Family Growth (NSFG) recueillies en 1995.

Le premier résultat de cette étude montre que l'inscription à temps plein par rapport à l'inscription à temps partiel augmente les probabilités de terminer les études collégiales. L'importance de ce facteur a amené Jacobs et Bertowitz King à tester leur modèle d'analyse séparément pour ces deux types d'inscription aux études. Ainsi, le niveau de scolarité de la mère ou du père n'influe pas sur l'obtention du diplôme collégial pour les deux groupes d'étudiantes (selon le type d'inscription). Toutefois, la race ou l'ethnie agit dans le sens attendu pour les étudiantes inscrites à temps plein, les probabilités de terminer leurs études étant plus faibles pour les nonblanches. Par ailleurs, l'appartenance raciale ou ethnique n'intervient pas chez les étudiantes inscrites à temps partiel.

Jacobs et Bertowitz King concluent leur analyse en précisant que leur modèle est beaucoup plus efficace pour expliquer les comportements aux études des étudiantes inscrites à temps plein. La probabilité d'obtenir un diplôme, pour les étudiantes à temps partiel, augmente si : a) elles ont été inscrites antérieurement au collège (previous spells of enrollment); $b$ ) elles ont terminé leurs études en été, ce qui suppose qu'elles ont eu accès à des cours donnés durant la période estivale. Leur probabilité de se retrouver dans la même situation diminue si : $a$ ) elles ont connu des délais entre la fin de leurs études secondaires et leur inscription aux études collégiales; $b$ ) elles travaillaient à temps plein au moment de leurs études collégiales (durant le mois observé). Les autres facteurs-les appartenances socioéconomique et raciale ou ethnique - ainsi que les statuts matrimonial et maternel des étudiantes

67. Ce sont des femmes hispaniques, afro-américaines en comparaison des femmes non - hispaniques blanches.

68. JACOBS, Jerry A. et BERKOWITZ KING, Rosalind (2002), « Age and College Completion : A Life-History Analysis of Women Aged 15-44», Sociology of Education, 75, 3, juillet : 211-230 (article 9).

69. " [...] the effect of timing measures, such as previous enrollment spells and delayed enrollment, on the likewood of completing a degree » (Jacobs et Berkowitz King, 2002: 212). 
Une critique féministe des théories néoclassiques du choix rationnel a montré que ces théories ne peuvent rendre compte des contraintes structurelles existantes pour les femmes comparativement aux hommes. inscrites à temps partiel ne différencient pas les étudiantes à temps partiel qui terminent leurs études collégiales de celles qui consacrent une période de temps à ces études sans les terminer. Jacobs et Bertowitz King mentionnent toutefois que d'autres études seront nécessaires afin de mieux comprendre les facteurs qui incitent ou empêchent les étudiantes et les étudiants à temps partiel de terminer leurs études.

\subsubsection{2 - La notion de capital humain contestée}

L'application des théories néoclassiques en économie de l'éducation, dont le concept du capital humain (à savoir que tous les jeunes, quelles que soient leurs appartenances sociales, formuleraient des attentes similaires par rapport à l'anticipation de gains en raison de la poursuite de leurs études et que l'estimation de gains supérieurs serait le facteur qui les stimulerait le plus à poursuivre leur scolarisation), est théoriquement problématique selon Irenee R. Beattie (2002). Celle-ci remet donc en question le modèle prédictif des théories du capital humain. Son étude ${ }^{70}$ est la première étape d'un processus de recherche en vue de déterminer comment le fait d'ignorer ces différences peut représenter un problème sérieux aux yeux des sociologues qui s'inspirent de ces théories néoclassiques. Par exemple, une critique féministe des théories néoclassiques du choix rationnel a montré que ces théories ne peuvent rendre compte des contraintes structurelles existantes pour les femmes comparativement aux hommes en situation d'emploi (ségrégation sexuelle des occupations et écarts salariaux malgré des diplômes obtenus dans les mêmes disciplines) ainsi que pour ce qui est de leurs responsabilités différenciées par rapport aux enfants (Beattie, 2002: 22). Des chercheuses et des chercheurs ayant mené des recherches sur les appartenances sexuelle (gender) et raciale ont formulé l'hypothèse que les femmes et les personnes de couleur peuvent vivre des expériences différentes de celles des hommes et de la population blanche relativement à leur processus de scolarisation et d'insertion au marché du travail (Beattie, 2002:22).

Beattie s'interroge en fait sur le modèle prédictif des théories du capital humain à partir de l'inclusion de diverses appartenances sociales dans ce modèle. La banque de données utilisée pour son analyse est celle de la High School and Beyond (HSB) du National Center for Educational Statistics (NCES) (1986) ${ }^{71}$. Des entrevues ont été réalisées en 1980 avec les élèves du secondaire (sophomore). Des relances auprès de ceux-ci et de celles-ci ont été faites en 1982, en 1984 et en 1986.

Les trois principaux sous-ensembles ou blocs de variables retenus sont: 1) des indicateurs du contexte socioéconomique des États ${ }^{72}$;2) des indicateurs des caractéristiques sociales des individus (Noir ou Noire ${ }^{73}$, Latino ou Latina ${ }^{74}$, et autres,

70. Irenee R. BEATTIE (2002), " Are All "Adolescent Econometricians" Created Equal? Racial, Class, and Gender Differences in College Enrollment », Sociology of Education, 75, 1, janvier : 19-43 (article 6).

71. Cette banque de données regroupe 11615 individus (5 649 hommes et 5966 femmes) ayant fréquenté des établissements d'enseignement secondaire privés ou publics (sophomore) en 1980. Seuls les élèves ayant obtenu leur diplôme d'études secondaires ont été retenus pour l'analyse.

72. Ces indicateurs sont mesurés par la scolarité et le sexe selon l'État; le revenu par personne, le taux de chômage et la moyenne du coût des études collégiales par État.

73. Cet élément est traité comme une variable unique dans son modèle d'analyse.

74. Cet élément est traité comme une variable unique dans son modèle d'analyse. 
statut socioéconomique de la famille, réussite scolaire (standardized test scores), attentes par rapport à l'éducation, nombre de sœurs et de frères plus âgés, statut matrimonial et présence d'enfants); et 3) des indicateurs du contexte de la communauté ${ }^{75}$. La variable dépendante est l'inscription des étudiantes et des étudiants aux études collégiales ou la poursuite des études au collégial ${ }^{76}$. Les appartenances de race et de classe sociale, les habiletés cognitives (standardized test scores) et le sexe constituent les principales variables témoins de l'étude de Beattie.

Beattie (2002) teste cinq modèles ${ }^{77}$ pour les jeunes hommes et deux pour les jeunes femmes ${ }^{78}$. Les résultats vont dans le sens des hypothèses formulées par la chercheuse. Il n'y a pas de modèle prédictif universel selon lequel l'inscription au collège serait liée pour toutes et tous à l'anticipation de gains monétaires supérieurs en raison de la poursuite d'études postsecondaires ou collégiales. Cependant, des variations s'observent selon les appartenances sociales et les habiletés cognitives.

Chez les jeunes hommes, chacune des principales variables témoins a un effet séparé sur la variable dépendante, la plus influente demeurant toutefois l'effet spécifique de l'appartenance raciale noire (modèles 2 et 5). L'impact des retombées monétaires de la poursuite d'études sur l'inscription au collège est plus important pour les étudiants non-noirs (blancs, latinos, asiatiques et autres). Ces différences dans les retombées entre les étudiants noirs et les autres varient également selon le contexte étatique. Les jeunes d'origine socioéconomique supérieure, tout comme ceux qui ont des habiletés cognitives élevées (réussite au secondaire), ne sont pas touchés dans leur inscription au collège par les retombées monétaires attendues. L'inverse s'observe pour ceux qui ont le moins bien réussi. Dans ce cas, leur perception des retombées monétaires escomptées influe sur leur inscription au collège, bien que, de façon générale, ils s'inscrivent moins au collège que les élèves ayant des habiletés élevées ou moyennes.

Pour les jeunes femmes, les résultats des analyses de régression logistique ${ }^{79}$ vont dans le sens des résultats observés chez les jeunes hommes. L'anticipation de retombées monétaires en raison de la poursuite de leurs études n'a pas d'effet significatif sur l'inscription des jeunes femmes au collège. Tout comme pour les jeunes hommes, certaines caractéristiques psychosociales et scolaires - un revenu élevé par personne dans leur État, des frais d'inscription scolaire plus bas, un statut socioéconomique familial supérieur, des résultats scolaires élevés, des attentes éducationnelles supérieures, le statut de célibataire, le fait de ne pas avoir d'enfants ou d'en avoir peu et la fréquentation d'une école secondaire privée - augmentent les proba-

75. Ces indicateurs sont la résidence en milieu urbain ou rural et la fréquentation d'une école secondaire privée.

76. Dans ces collèges, les études ont une durée de deux ou de quatre ans.

77. Le modèle 1 inclut la mesure directe des retombées de la poursuite d'études pour chacun des indicateurs des sous-ensembles ou blocs de variables (state-level economic context individual background, schoolcommunauty context) sur l'inscription au collège. Le modèle 2 ajoute au modèle 1 l'interaction de l'appartenance raciale. Le modèle 3 retient, en plus des variables du modèle 1, I'interaction de l'appartenance de classe (origine socioéconomique et coût des études). Le modèle 4 ajoute au modèle 1 l'interaction de la réussite scolaire au secondaire (standardized test scores). Enfin, le modèle 5 regroupe l'ensemble des modèles précédents.

78. Les modèles 1 et 5 sont retenus dans ce cas.

79. Cela vaut pour le modèle 1 . 
bilités de s'inscrire au collège (Beattie, 2002 : 33). Toutefois, contrairement aux jeunes hommes, les probabilités de s'inscrire au collège sont plus élevées pour les jeunes femmes de descendance africaine ou latino-américaine et qui vivent dans des États où le taux de chômage est plus élevé.

L'application du t-test à ces résultats, pour mesurer l'effet de cette analyse séparée selon le sexe sur les résultats obtenus, permet de montrer à nouveau qu'il n'y a pas de différence entre les sexes concernant les retombées monétaires anticipées par leur scolarisation supérieure. Le coût des études a toutefois plus d'impact négatif sur les jeunes femmes que sur les jeunes hommes, bien que les premières s'inscrivent en moyenne en plus grande proportion que les seconds au collège. Les filles de la classe sociale supérieure s'inscrivent davantage que les jeunes hommes du même groupe social. Les femmes noires et, de façon plus modeste, les femmes latinoaméricaines s'inscrivent au collège en plus grande proportion que les femmes blanches. Les jeunes femmes retirent toutefois moins de bénéfices de la fréquentation des écoles privées que les jeunes hommes sur leur probabilité de s'inscrire au collège.

Pour ce qui est du modèle 5, lequel inclut l'ensemble des facteurs à l'étude, les variables interactives (race, statut socioéconomique, standardized test scores) ne changent pas le sens des relations, comme cela avait été le cas pour les jeunes hommes. Pour Beattie, cela signifie que les femmes, quels que soient leur appartenance sociale et leurs résultats scolaires, sont moins sensibles aux retombées monétaires anticipées dans la décision de poursuivre leurs études collégiales ${ }^{80}$.

De son côté, P. Duraisamy (2002) inscrit également son étude ${ }^{81}$ à l'intérieur du paradigme du capital humain. Cet auteur précise toutefois que les rendements positifs de la scolarisation aux divers ordres d'enseignement dans les pays en voie de développement ne sont pas que d'ordre pécuniaire, en particulier pour les femmes dans le contexte indien ${ }^{82}$.

Les objectifs de Duraisamy dans son étude sont d'estimer, pour les années 19831994, le rendement privé de la scolarisation (private returns to education) des travailleurs et des travailleuses qui occupent un emploi salarié83 (wage employment)

80. " This finding suggests that not only are young women less responsive to returns to schooling than young men, but that the effect of returns does not vary by race, class, cognitive ability, or cost [des études] for young women as it does for young men. Other variables remain essentially unchanged [pour les femmes] from those in Model 1 » (Beattie, 2002: 33).

81. DURAISAMY, P. (2002), " Changes in Returns to Education in India, 1983-94: By Gender, Age-cohort and Location ", Economics of Education Review, 21, 6: 609-622 (article 8).

82. DURAISAMY, P. et MALATHY, R. (1990), "Impact of Public Programs on Fertility and Gender Specific Investment in Human Capital of Children in Rural India : Cross Sectional and Time Series Analysis », dans T.P. Schultz (dir.), Research in Population Economics, vol.7: 157-186, CT, États-Unis : Jai Press Inc.; R. MALATHY (1994), "Education and Women's Time Allocation to Nonmarket Work in an Urban Setting in India ", Economic Development and Cultural Change, 42, 4: 743-760 cités dans Duraisamy (2002: 610).

83. Les travailleuses et les travailleurs permanents salariés sur qui porte cette étude ne représentent respectivement que 6,2 \% et 16,7\% de l'ensemble de la main-d'œuvre aux Indes en 1993-1994. Ces pourcentages n'ont alors à peu près pas augmenté par rapport à 1983 (5,4\% de femmes et $18,1 \%$ d'hommes). La participation au travail (self-employed, regular-wage/ salaried workers, casual laborers) était de 29,5\% et de 28,3\% pour les femmes en 1983 et en 1993-1994; celle des hommes se situait à 53,8\% et à 54,4\% (Duraisamy, 2002: 611). 
selon le sexe, le groupe d'âge ${ }^{84}$ et la résidence ${ }^{85}$ ainsi que d'évaluer les changements de ces retombées pour la période 1983-1994. L'auteur poursuit en outre dans sa recherche des fins méthodologiques ${ }^{86}$. Les données sur lesquelles porte cette recherche pour les années 1993-1994 sont représentatives de la population à l'échelle nationale. Les données sur l'emploi sont tirées de deux enquêtes menées par le National Sample Survey (NSS) en 1983 et en 1993-1994²7. La variable dépendante principale est la moyenne de salaire quotidien ${ }^{88}$. Deux variables indépendantes nominales mesurent la scolarisation : a) les années ${ }^{89}$ additionnelles de fréquentation d'un ordre d'enseignement par rapport au précédent (primaire, échelon moyen (middle), secondaire, deuxième cycle du secondaire (higher secondary), collégial ou universitaire $^{90}$ ); et b) l'obtention ou non d'un diplôme ou d'un certificat technique. D'autres variables et méthodes de calcul entrent aussi en jeu dans le modèle.

Les principaux résultats de l'étude sont les suivants. L'un des bénéfices de la scolarisation ou de l'éducation formelle est le fait, pour un individu, d'augmenter ses possibilités d'entrer sur le marché du travail permanent salarié (regular wage work), et ce, encore plus pour les années passées en enseignement supérieur, en particulier pour les personnes qui ont fréquenté les collèges. Pour celles qui occupent un emploi salarié, le taux de rendement privé de la scolarisation augmente jusqu'au secondaire et décline par la suite. Les personnes titulaires d'un diplôme ou d'un certificat technique bénéficient toutefois d'un taux de rendement supérieur à celui des personnes qui ont fréquenté le collégial.

Le taux de rendement privé de la scolarisation est plus élevé pour les femmes que pour les hommes à l'échelon moyen, au secondaire ${ }^{91}$ et au deuxième cycle du secondaire (higher secondary level). Les cohortes les plus jeunes (15-29 et 30-44 ans) comparativement à la cohorte la plus âgée (45-65 ans) bénéficient moins d'années scolaires additionnelles au primaire, à l'échelon moyen et au secondaire. Dans le cas du collégial ou de l'obtention d'un diplôme ou d'un certificat technique, la rentabilité est cependant plus grande pour la cohorte des 15-29 ans. Le taux de rendement privé des études (primaire, secondaire et obtention d'un diplôme ou d'un certificat technique) est supérieur pour les personnes résidant en milieu rural ${ }^{92}$. À l'inverse, les

84. Les trois groupes retenus sont les 15-29 ans, les 30-44 ans et les 45-65 ans.

85. Le lieu de résidence peut être en milieu rural ou en milieu urbain. Duraisamy rappelle que très peu d'études dans les pays en voie de développement ont tenu compte de cette diversité résidentielle, la majorité des recherches ne retenant que la résidence urbaine et ses liens avec la scolarisation.

86. Cela concerne la mesure de ces rendements et des biais provenant de la sélection des échantillons.

87. Cette dernière enquête a touché environ 69230 personnes vivant en milieu rural et 46179 personnes en milieu urbain (urban households) de 7284 villages et 4792 quartiers urbains (urban blocks) et environ le même nombre de personnes en 1983.

88. L'expression en anglais est la suivante: « logarithm of the daily wage rate » (Duraisamy, 2002: 613).

89. L'information sur le nombre d'années de scolarité par individu n'étant fournie, il est assumé dans le modèle qu'un individu passe respectivement 5, 3, 2, 2 et 3 ans pour terminer sa scolarité à partir du primaire jusqu'à la fin du collège (Duraisamy, 2002: 613).

90. Les personnes illettrées et celles qui n'ont pas achevé leurs études primaires constituent le groupe de référence.

91. C'est le cas en particulier au secondaire, où le taux de rendement privé, pour des années supplémentaires de scolarisation, est plus de deux fois supérieur à celui des hommes (Duraisamy, 2002: 621).

92. Duraisamy qualifie ce résultat de " striking finding » (p. 621) qui justifie l'inclusion de la résidence en milieu rural et non seulement en milieu urbain dans les modèles des études menées dans des pays en voie de développement sur le rendement de la scolarisation. 
taux de rendement privé des études du deuxième cycle du secondaire et du collégial sont plus élevés en milieu urbain. À la campagne ou à la ville, les taux de rendement privé pour les femmes sont supérieurs à ceux des hommes, et ce, pour l'échelon moyen, le secondaire et le deuxième cycle du secondaire.

De façon générale, un changement considérable s'observe à travers la période considérée, particulièrement dans le cas des femmes. Pour ces dernières, les taux de rendement privé ont baissé de 1983 à 1993-1994 concernant le primaire et l'échelon moyen. Ils ont toutefois augmenté pour le secondaire ${ }^{93}$ et le collégial. Les taux de rendement global (absolute returns) à l'échelon moyen et au secondaire sont plus élevés pour les femmes que pour les hommes dans l'ensemble des périodes étudiées. À l'inverse, le taux de rendement global des hommes pour l'obtention d'un diplôme ou d'un certificat technique a augmenté au cours de la période 1993-1994.

\subsubsection{3 - Les politiques institutionnelles et le pouvoir hégémonique}

Dans la présente section, les articles portent sur les enjeux liés aux distinctions de classe sociale, de race et de sexe dans les établissements d'enseignement supérieur. Ces études montrent la façon dont des discours et des pratiques entourant le processus d'admission aux programmes prestigieux de médecine (Borst, 2002) et de droit aux États-Unis, les examens aux universités d'Oxford et de Cambridge en Angleterre (Deslandes, 2002) et la contestation des mesures de redressement (affirmative action) aux États-Unis (Solórzano et Yosso, 2002) peuvent servir de mécanismes aux élites (classe élevée et moyenne-supérieure, blanche, masculine) pour maintenir leur hégémonie. Les études historiques rappellent l'ancrage du pouvoir de ces élites sur une longue période de temps et la manière dont, à travers des institutions prestigieuses (universités, Cour suprême), elles ont imposé l'adoption de leurs normes culturelles et de leurs pratiques aux autres groupes sociaux.

L'expansion de l'enseignement supérieur aux États-Unis durant les années 20 et 30 a radicalement changé le profil des étudiants dans les écoles de médecine. Cette expansion a profité à des hommes dont les origines sociales étaient radicalement différentes de celles des Américains qui occupaient jusqu'à cette époque les professions libérales ${ }^{94}$. Pour Charlotte G. Borst (2002), cette diversité étudiante ne faisait toutefois pas consensus. Dans une étude ${ }^{95}$ critique, historique et qualitative des discours entourant les débats et les décisions par rapport au processus d'admission dans les écoles de médecine aux États-Unis de 1920 à 1950 science avec les catégories analytiques de race et de sexe (gender). À son avis, l'analyse, qui porte principalement sur l'histoire de la progression et du déclin de l'utilisation de tests standardisés dans le processus d'admission, illustre les interactions

93. Aux fins de cette comparaison dans le temps, le secondaire et le deuxième cycle du secondaire ont été regroupés dans la même catégorie.

94. "There were far more men who came backgrounds radically dissimular to that of the "old American stock" that had usually populated the professions " (Borst, 2002: 183).

95. Charlotte G. BORST (2002) «Choosing the Student Body: Masculinity, Culture, and the Crisis of Medical School Admissions, 1920-1950 », History of Education Quartely, 42, 2, été: 181-214 (article 5).

96. Mentionnons que l'étude de Dyhouse (2002) en Angleterre, examinée au point 2.2.1.1, portait à peu près sur la même période que celle de Borst (2002). 
complexes de la science, de son institutionnalisation à l'intérieur des universités ainsi que des normes culturelles entourant le sexe et la race. Cette dernière devient un enjeu social et culturel à la fin du XIX ${ }^{\mathrm{e}}$ siècle et provoque ce que des historiens (Rotondo, 1993; Kimmel, 1997 cités dans Borst, 2002: 185) ont désigné comme une «crise » de l'identité masculine chez les hommes nés aux États-Unis dont les ancêtres avaient émigré de l'Europe du Nord. Cette «crise» se serait accentuée au cours du $\mathrm{XX}^{\mathrm{e}}$ siècle avec l'arrivée des femmes sur le marché du travail et leur entrée au collège, de même qu'avec la concrétisation des espoirs de mobilité sociale d'autres groupes ethniques par leur fréquentation des établissements d'enseignement supérieur (Borst, 2002: 185).

Les données sur lesquelles porte l'analyse de la chercheuse proviennent du dépouillement des archives ${ }^{97}$ de la American Medical Association (AMA), de la Association of American Medical Colleges (AAMAC), de celles des doyens de ces collèges ou écoles de médecine et d'autres acteurs institutionnels ${ }^{98}$, de même que sur des analyses d'ouvrages scientifiques.

Le contrôle du processus d'admission par les élites médicales s'inscrit dans une logique hégémonique d'un groupe social sexuel et ethnique particulier. À travers son étude, Borst montre que les représentations qu'elle dégage des argumentaires des discours entourant les débats, les décisions et les pratiques d'admission ${ }^{99}$ des étudiants en médecine ont pour objet de maintenir l'hégémonie de ce prototype idéal de l'Américain de souche (old American stock), de «bonne» origine (right background), les autres étant les "mauvais hommes" (wrong men). Borst montre en outre que les individus acteurs qui sont favorables à cette visée et à cette pratique hégémonique oscillent entre un argumentaire basé sur la science et l'importance de mesures et de critères objectifs ${ }^{100}$, lorsque les résultats de leur utilisation dans la sélection des étudiants en médecine favorisent le statu quo, et un argumentaire fondé sur des mesures et des critères subjectifs difficilement mesurables (quality of mind, brains, character, adaptability, social fitness and motivations), lorsque les hégémonies raciale et sexuelle ne sont plus assurées.

Les pratiques de sélection subissent en outre ces influences. Ainsi, l'entrevue individuelle et les quotas s'ajoutent aux pratiques antérieures à certaines périodes au cours des années 20 à 50, dans le but, selon ces protagonistes, de contrer les effets pervers de mesures objectives favorisant l'entrée des "mauvais hommes ", c'est-àdire les Juifs, les Italiens, les Catholiques et les Afro-Américains. La chercheuse indique toutefois que les groupes visés ou rejetés dans l'application de ces pratiques n'ont pas toujours été les mêmes durant la période étudiée. Des historiens ont noté que les attitudes au sujet de la race, mais non du sexe cependant, ont changé après

97. Cela regroupe les rapports, la base de données statistiques, la correspondance et les résolutions des comités exécutifs, ces derniers étant composés principalement des doyens des collèges ou écoles de médecine universitaires

98. C'est le cas notamment de l'American Council on Education et d'autres acteurs.

99. Ces débats n'aboutissent pas toujours à des consensus. Il en est de même des pratiques, qui ne sont pas toujours uniformes dans les écoles de médecine malgré les décisions de l'AAMAC.

100. Pensons notamment au recours à des critères d’admission basés sur les antécédents scolaires (réussite des apprentissages scolaires dans les programmes scientifiques, etc.). 
la Seconde Guerre mondiale, certains groupes, les Juifs et d'autres groupes immigrants de l'Europe de l'Est et du Sud étant considérés à partir de cette époque comme des «Blancs» et les wrong men devenant principalement les Afro-Américains (Borst, 2002: 211).

La chercheuse montre de plus, principalement dans le contexte des débats durant les années 20 à 50 entourant le développement de tests standardisés et de leur utilisation comme critère unique ou comme un des critères de sélection, que les décisions et les pratiques qui en ont découlé ont eu un effet réel sur l'entrée des femmes et des minorités ethniques dans les écoles de médecine aux États-Unis et dans la profession médicale. En 1920, moins de $5 \%$ des femmes font partie de l'ensemble du corps médical aux États-Unis. Ce nombre va augmenter légèrement durant la Seconde Guerre mondiale. Toutefois, la progression des femmes ne sera pas importante jusqu'aux années 70 (Borst, 2002: 184). Le nombre d'étudiantes en médecine va suivre la même tendance, bien qu'il soit déjà plus élevé que pour les Afro-Américains. En 1938-1939, 1144 femmes étudient en médecine, soit 5,4 \% de la population étudiante ${ }^{101}$. Les universités (écoles de médecine), soit Harvard, Jefferson, Georgetown, Saint-Louis, Darwouth, Hannemann et Emory, n'acceptent pas de femmes avant la fin de la Seconde Guerre mondiale ${ }^{102}$. Durant l'année scolaire 1938-1939, seulement 350 Noirs étudient en médecine, soit 1,64 \% de la population étudiante ${ }^{103}$. En 1955, 236 étudiants noirs sont inscrits à des écoles traditionnellement fréquentées par les Blancs, la majorité, soit 761, se dirigeant encore vers les deux écoles des universités fréquentées par les Noirs (Borst, 2002 :189). Dans le cas des étudiants juifs, en 1928, le Collège des médecins et chirurgiens de l'Université Columbia a ramené ( $h a d$ cup) à $20 \%$ le taux d'inscription des étudiants juifs, alors qu'il était de $50 \%$ en 1923 (Borst, 2002: 194-195).

Durant la seconde moitié du XXe siècle, poursuit Borst, les enjeux sociaux au sujet des identités masculine et blanche et de classe moyenne se sont interposés dans les normes américaines de succès ${ }^{104}$, lesquelles étaient de plus en plus fortement dépendantes pour l'accès à l'enseignement supérieur et à ces programmes prestigieux. En conclusion, Borst montre que ces débats, sur les politiques d'admission et l'entrée des femmes et de certains groupes raciaux ou ethniques dans les établissements d'enseignement supérieur et leur inscription à des programmes élitistes, se poursuivent encore de nos jours. Mis en place durant les années 70 en vue de favoriser principalement l'accessibilité des femmes et des Noirs, les mesures de redressement (affirmative action) ont été moins nombreuses ou ont perdu de leur

101. En 1938-1939, 100 de ces étudiantes sont inscrites au Women's Medical College of Pennsylvania (WMCP), école non mixte, la majorité étant inscrites dans des collèges mixtes (Borst, 2002 : 189).

102. Martha Tracey, doyenne (1938), " Report of a Conference on Opportunity for Women in the Medical Profession and the Selection of Medical Students ", Women's Medical College of Pennsylvania Archives cité dans Borst (2002: 189)

103. Seulement 98 d'entre eux poursuivaient leurs études dans des écoles traditionnellement fréquentées par les Blancs, les autres se dirigeant vers les universités noires (Meharry ou Howard University) (Borst, 2002: 189).

104. Ce sont les normes de succès en fait de statut social (économique, politique, culturel). 
poids dans le processus d'admission ${ }^{105}$. Cependant, Borst garde un certain espoir d'une plus grande mixité à partir de la prise de position publique, en 1998, du président de l'AAMC, le docteur Jordan Cohen, en faveur d'une véritable diversité des médecins, celui-ci argumentant que celle-ci est nécessaire pour assurer aux ÉtatsUnis des soins de qualité élevée ${ }^{106}$.

Examinons maintenant l'article ${ }^{107}$ de Daniel G. Solórzano et Tara J. Yosso (2002) qui porte sur les questions de la race, du racisme et des mesures de redressement (affirmative action). Cette recherche permet de faire des liens avec des hypothèses formulées par Borst (2002) à la fin de son article sur la diminution de mesures de rattrapage et de la perte de leur importance dans le processus d'admission aux écoles de médecine aux États-Unis. La même problématique vaut également pour d'autres programmes prestigieux, en particulier ceux de droit. J'ai classé cet article de Solórzano et Yosso (2002) dans le quatrième groupe (voir l'annexe II). Les approches féministes constituent un des cadres d'analyse dans le développement épistémologique de la théorie critique radicale ${ }^{108}$ (critical theory radical ou CTR). Plusieurs références, dont des écrits de Solórzano et Yosso, traitent du genre (gender). Néanmoins j'ai classé cet article dans le quatrième groupe du fait qu'il porte principalement sur l'appartenance raciale.

Dans leur article, Solórzano et Yosso traitent des enjeux concernant l'équité, l'accès et des mesures de redressement en enseignement supérieur aux États-Unis à partir du paradigme et de la méthode analytique de la théorie critique radicale. Ce cadre d'analyse peut être utilisé pour théoriser et examiner comment la race et le racisme ont de multiples répercussions sur les structures, les processus et les discours dans le contexte de l'enseignement supérieur (Solórzano et Yosso, 2002 : 164). Solórzano et Yosso, qui enseignent à l'Université de Californie, situent la production de leur article dans le contexte particulier de la Proposition 209, adoptée en Californie en 1997, laquelle met fin dans cet État à des pratiques limitées de mesures de redressement (affirmative action) basées sur la race ${ }^{109}$. À leur avis, l'adoption de cette loi a eu des effets en Californie et sur le plan national concernant la décroissance proportionnelle des étudiantes et des étudiants américains, africains, latinos et autochtones, hypothèse partagée par Borst (2002).

Solórzano et Yosso analysent aussi le discours légal et les enjeux qui en découlent. Les deux créent dans leur article une « contre-histoire à multiples facettes » ( $a$ multilayered counterstory) afin de contextualiser le 6th Circuit Federal Case en enseignement supérieur, appelé l'«affaire Grutter v. Bollinger (1997)», soumise récemment à

105. " By the fall of 2000, most universities had dropped overt affirmative action policies for admissions, and minority applications to medical schools have declined by 12 percent since 1996. AAMC News Room, Issues Summaries, 'Diversity'.", December 7, 1999 » (Borst, 2002 : 214).

106. " $[A]$ diverse health professions workforce was necessary in order for the United States to continue to deliver high-quality health care » (Borst, 2002: 214).

107. SOLÓRZANO, Daniel G. et YOSSO, Tara J. (2002). "A Critical Race Counterstory of Race, Racism, and Affirmative Action ", Equity \& Excellence in Education, 35, 2, mai : 155-168 (article 10).

108. Cette théorie constitue le cadre d’analyse principal de Solórzano et Yosso.

109. " [...] limited " race-based" affirmative action » (Solórzano et Yosso, 2002: 164). 
la Cour suprême ${ }^{110}$ des États-Unis. La plaignante est Barbara Grutter, femme blanche ayant fait une demande d'admission en 1996-1997 sans succès à la Faculté de droit de l'Université du Michigan. Grutter avance que, dans ses politiques d'admission en faveur de la race, cette université établit une discrimination contre des étudiants qui, comme elle, sont plus qualifiés ${ }^{111}$. Le défenseur est Lee Bollinger, ancien président de l'Université du Michigan et ancien doyen de l'École de droit de la même université. La grande particularité de cette affaire est la présence, comme tierce partie, des étudiants (student intervenors ${ }^{112}$ ), ce qui est une pratique rare dans de telles causes ${ }^{113}$.

La procédure d'écriture de cet article scientifique et la méthode d'analyse pratiquée sont celles de la counterstory-telling ${ }^{114}$, technique ayant une longue tradition, selon le chercheur et la chercheuse, en sciences sociales et humaines de même qu'en droit, comme méthode de la théorie critique radicale élaborée aux États-Unis durant les années 80 et 90 . Cette méthode permet de donner la parole aux personnes qui vivent en marge de la société et de mettre en doute la parole des personnes au pouvoir.

Solórzano et Yosso utilisent quatre sources dans l'écriture de leur récit: 1) leurs données et rapports de recherche réalisés en 1999-2000 sur le climat racial au campus de l'University of Michigan ${ }^{115}$, à l'École de droit de la même université ${ }^{116}$ et à l'University of California, Berkeley ${ }^{117}$; 2) les écrits en sciences sociales et humaines sur les questions d'équité, d'accès et de traitement en éducation de même que sur les litiges en éducation, principalement les cas soumis à la Cour suprême des États-Unis depuis l'affaire Bakke $e^{118}$; 3) leurs expériences professionnelles; et 4) leurs expériences personnelles.

Les trois personnages (fictifs-réels) de leur histoire sont Claudia ${ }^{119}$, le juge Thurgood Marshall ${ }^{120}$ et Ruby Puentes ${ }^{121}$. L'histoire commence avec Claudia entrant à la Cour suprême pour entendre l'affaire Grutter v. Bollinger en janvier 1991. Elle s'assoit, avec l'intention de prendre des notes, lorsqu'elle se rend compte que le juge Thurgood Marshall et Ruby Puentes se trouvent à ses côtés. L'histoire porte sur leurs

110. Au moment de la publication de l'article de Solórzano et Yosso, le jugement n'avait pas été rendu dans cette affaire.

111. " [...] that the University of Michigans's race conscious admissions policy discriminated against " more qualified" white applicants like herself » (Solórzano et Yosso, 2002: 155).

112. L'argumentation de ce groupe dans cette affaire va dans le sens souhaité par les trois personnages de la counterstory-telling.

113. Solórzano et Yosso (2002: 155).

114. Cette méthode et cette procédure d'écriture ont aussi une fonction pédagogique. Voir Solórzano et Yosso (2002: 156).

115. W. Allen et D.G. Solórzano (2000, octobre) relativement à l'affaire Gratz et al. v. Bollinger et al.

116. W. Allen et D.G. Solórzano (2000, septembre) relativement à l'affaire Grutter et al. v. Bollinger et al.

117. D.G. Solórzano et W. Allen (2000, août) relativement à l'affaire Castañeda et al. v. UC Regents et al.

118. Regents of University of California v. Bakke (1978).

119. Le personnage de Claudia représente une avocate, Chicana, spécialiste des droits civils et professeure à I'University of California.

120. Le juge Marshall personnifie le juge qui a siégé à la Cour suprême des États-Unis et qui est connu pour ses prises de position contre le racisme, notamment dans un jugement dissident rendu avec trois juges dans l'affaire Bakke. Le juge Marshall a été notamment l'avocat (attorney) du NAACP dans l'affaire Brown v. Board of Education en 1954 (Solórzano et Yosso, 2002 : 157, 161).

121. Ruby Puentes (la traduction anglaise de Puentes est Bridges) représente Ruby Bridges, la petite fille Afro-Américaine, qui, au cours des années 50, à la suite de l'ordre de la Cour fédérale sur la déségrégation raciale scolaire, avait été escortée par quatre policiers fédéraux de La Nouvelle-Orléans à une école primaire fréquentée exclusivement par des jeunes filles blanches (Solórzano et Yosso, 2002: 157). 
dialogues concernant les enjeux de cette affaire et sa mise en contexte avec la jurisprudence des autres litiges concernant la race et le racisme dans le domaine de l'éducation aux États-Unis.

Il est très difficile, pour bien rendre justice à cette procédure particulière d'écriture et à cette méthode d'analyse, de s'en tenir uniquement à la présentation des principaux résultats de cette recherche. Notons que l'argumentaire des personnages est basé sur les résultats des études en sciences sociales par rapport à cette problématique. Toutefois, j'énonce ci-dessous quelques-uns des grands enjeux, fondements théoriques et idéologiques des argumentations avancées par Claudia, Ruby Puentes et le juge Marshall :

- D'abord, selon le juge, les mesures de redressement (affirmative action) en tant que politique ayant des objectifs à atteindre en enseignement supérieur durant une période de temps déterminée ont été de courte durée, soit de 1968 à 1978;

- La race est un construit social. Le concept de race a été principalement utilisé pour différencier les peuples avec pour objectif d'établir une discrimination entre eux (discriminated against them);

- $\quad$ Les concepts de race et de racisme ne peuvent être séparés (J. Calmores (1997) cité dans Solórzano et Yosso (2002 : 158)). Le racisme, selon Manning Marable ${ }^{122}$ (1992), est un système d'ignorance, d'exploitation et de pouvoir utilisé pour opprimer les Afro-Américains, les Latinos, les Asiatiques, les Américains du Pacifique, les Indiens-Américains et d'autres peuples sur la base de l'ethnicité, de la culture, des manières (mannerisms) et de la couleur;

- Le fait d'être blanc ou blanche a une valeur de propriété ${ }^{23}$ (whiteness actually has property value ${ }^{124}$ ), dans les sens économique, politique et culturel;

- La qualité de l'éducation a toujours été jugée en fonction de faire participer les Noirs, les Latinos et d'autres minorités à l'école des Blancs, et non l'inverse, les déficits culturel et scolaire étant toujours évalués du même côté avec des variantes historiques sur le ou les groupes raciaux jugés déficitaires;

- La permanence du racisme (Bell, 1992), ou le racisme sytémique, n'a pas encore été reconnue par la jurisprudence en matière d'éducation ${ }^{125}$;

- L'argumentation dans les plaidoyers est aussi faite sur la prétention que la race est une affaire personnelle, privée. Le besoin de mesures de redressement pour remédier au racisme n'a pas encore été reconnu dans la jurisprudence. Il importe toutefois, selon les trois personnages de la counterstory, de rappeler que la mesure d'affirmative action n'est qu'un des moyens pour contrer le racisme et ne peut être le seul moyen envisagé dans une société où le racisme fait système, en particulier dans le domaine de l'éducation pour plusieurs groupes sociaux. Il en est de même de la déségrégation scolaire (Brown v. Board of Education,

122. Ces références et celles qui suivent sont citées dans le texte de Solórzano et Yosso (2002).

123. On y fait aussi mention ailleurs dans le texte des privilèges des hommes (Solórzano et Yosso,2002: 160, 162).

124. Solórzano et Yosso (2002: 158).

125. C'est le cas notamment dans les affaires Gratz v. Bollinger (1997) et Bakke v. Regents of University of California (1978). Dans cette dernière affaire, le juge Powell réinterpète le concept de « discrimination raciale » comme faisant référence à toute race ou ethnicité, y compris celle des Blancs (p. 163). 
1954). Celle-ci n'a pas garanti, après le jugement de la Cour, en tant que mesure unique en éducation, une mixité et une égalité raciales de traitement (Orfield et Eaton, 1996);

- Les tentatives des écoles qui veulent ajouter des programmes spéciaux, «enrichis », ont pour objet de " garder » les élèves blancs à l'école publique et non d'y intégrer d'autres groupes raciaux;

- La tentative qui consistait à fixer un nombre précis pour assurer une certaine équité raciale (racial tipping point) dans les écoles, pratique des années 60 et 70, est maintenant envisagée comme nuisible. Le maintien de la définition de ce concept uniquement en termes numériques comporte certains dangers s'il est dissocié d'autres caractéristiques, dont celles du pouvoir ou du prestige d'un groupe. Par exemple,, les Blancs (au pouvoir), comme dans le cas de la Californie, argumentent qu'ils sont devenus une minorité numérique et qu'ils ont besoin de ce fait d'être protégés;

- Le concept de racial tipping point doit être traité comme le concept de diversité, et séparément du concept de pluralisme ${ }^{126}$;

- Une plus grande diversité raciale devrait être un objectif visé des politiques universitaires d'admission. Cette diversité ne doit pas se manifester uniquement dans les politiques d'admission en enseignement supérieur, mais également dans les programmes d'études et la pédagogie et elle doit être reconnue comme partie intégrante de la vie universitaire. Cette diversité profiterait à l'ensemble des étudiants des campus.

Élaborée par Bell (1987), la théorie de la convergence des intérêts (interestconvergence theory) aide à comprendre, selon Solórzano et Yosso, l'adoption récente de la Proposition 209, laquelle met fin aux pratiques "limitées" de mesures de redressement concernant à la race dans le domaine de l'éducation. À la suite de leur analyse sociale comparative du discours légal, Claudia, Ruby Puentes et le juge Thurgood Marshall ne peuvent s'empêcher de rappeler en conclusion la nécessité de l'éducation dans la prise de conscience des inégalités et de l'empowerment pour les gens de couleur ${ }^{127}$.

Sur un autre thème, retournons dans l'histoire, cette fois durant la période 18501920 en Angleterre pour y mesurer combien la tradition naissante des examens et de leur réussite, aux universités d'Oxford et de Cambridge, était associée aux enjeux d'identités de classe et de sexe. Paul R. Deslandes (2002) tente dans son étude ${ }^{128} \mathrm{de}$ circonscrire les significations culturelles et sexuelles (gendered) des examens pour les étudiants et étudiantes de premier cycle. À cette fin, il analyse la forme et le contenu des examens à partir principalement de l'expression des étudiants ${ }^{129}$. Ses données

126. Le pluralisme est défini trop souvent, selon Lawrence (2001) cité dans Solórzano et Yosso (2002: 159) en faveur de la culture et du mode de vie des Blancs.

127. "The elimination ef exposing and dismantling white privilege begins by educating and empowering people of color » (Solórzano et Yosso, 2002: 164).

128. DESLANDES, Paul R. (2002). " Competitive Examinations and the Culture of Masculinity in 0xbridge Undergraduate Life, 1850-1920 », History of Education Quarterly, 42, 4, hiver : 544-578 (article 7).

129. Le discours des étudiantes est aussi analysé dans la dernière section de son article. 
sont puisées dans 556 magazines et journaux étudiants et périodiques, dans des publications officielles des deux universités et dans des ouvrages scientifiques.

Les examens officiels, associés au prestige académique retrouvé des anciennes universités, ont été introduits tôt au XIX ${ }^{\mathrm{e}}$ siècle. Durant la seconde moitié de ce siècle, la régulation de ce processus s'instaure (Deslandes, 2002: 551). On nomme les examens tripos (or honors) examinations à Cambridge et final schools à Oxford ${ }^{130}$. Les examens, leurs finalités et principalement leurs significations s'inscrivent, selon Deslandes, dans un contexte plus large de la montée ou de la progression de la société professionnelle en Angleterre. Ce contexte a influé profondément sur les façons dont les crédits (credentials) ont été mesurés à Oxbridge ${ }^{131}$, et la réussite aux examens a constitué un nouvel enjeu pour les familles et les jeunes de classe moyenne-supérieure afin d'avoir accès aux positions de pouvoir dans la société anglo-saxonne.

Deslandes mentionne en outre un second enjeu, encore plus important à son avis, soit celui des identités sexuées ou de la masculinité dans cette société «professionnelle» en progression. Enjeu masculin lié au fait récent que les femmes, admises dans ces deux universités en 1870, dans des collèges féminins séparés de ceux qui étaient occupés par les jeunes hommes, ont obtenu en 1880 à Oxbridge, à la suite de leurs revendications, de pouvoir passer les examens (to sit examinations). Elles n'étaient toutefois pas autorisées officiellement à obtenir un diplôme (to formally take degrees). Dans ces contextes d'enjeux de distinctions de classe et de sexe, les constructions symboliques et les significations des jeunes par rapport aux examens prennent tout leur sens. C'est précisément cet aspect des examens que le chercheur tente d'approfondir. Il approche principalement ces significations sous l'angle des rites de passage menant à des identités culturelles et de genre (gender).

Les principales conclusions de Deslandes sont que ces examens, en tant que premiers moyens de réussite scolaire (academic achievement) et de qualification professionnelle pour les positions de pouvoir, notamment dans les affaires de l'État et dans l'Empire colonial de l'Angleterre, ont acquis durant la période 1850-1920 un vaste ensemble de significations. Celles-ci illustrent comment ces exercices étaient signifiants pour les étudiants de premier cycle durant cette période et qu'ils constituaient des enjeux pour eux, d'où leur intérêt, par l'entremise des magazines et journaux étudiants, à faire monter les enchères pour ceux qui en sortiraient vainqueurs ${ }^{132}$.

En employant un langage, des images et des métaphores qui «construisent » les examens comme «des épreuves horribles, des tests de caractère et des rituels mas-

130. L'Université de Cambridge offrait davantage des programmes de sciences, principalement de mathématiques, tandis que l'Université d'0xford donnait des programmes de sciences humaines et sociales.

131. Cette expression est employée pour parler à la fois des universités d'0xford et de Cambridge.

132. La préparation aux examens et ses nombreux rituels ont joué un rôle également, selon Deslandes, en tant que rites de passage qui marquent la transition masculine étudiante de l'enfance à la maturité (from boyhood to manhood), de membre d'une nouvelle élite professionnelle ayant partagé des expériences communes. 
culins sacrés ${ }^{133}$ ", les étudiants réussissent à créer et à répandre une culture d'extrême compétition et de lutte qui leur permet de définir (to cast) les vainqueurs (aux examens) en des termes superbement masculins ${ }^{134}$. Grâce à l'emprunt d'une rhétorique exagérée pour décrire les examens, cette stratégie aide ces derniers, poursuit le chercheur, à définir ces activités comme des activités masculines. La tendance en question est encore plus importante avec l'entrée des femmes à Oxbridge caractérisée alors par ces jeunes hommes comme une université assiégée par les femmes ${ }^{135}$.

Deslandes observe des convergences dans les discours des jeunes femmes par rapport aux jeunes hommes pour ce qui est de leurs représentations des examens en tant que rite de passage à la vie adulte et par rapport à la rhétorique empruntée d'anxiété et d'animosité à l'égard des examens. Il note cependant un plus grand nombre de divergences. Ainsi, les jeunes femmes, étudiantes à Oxbridge, mettent davantage l'accent sur les retombées positives de la promotion collective des femmes plutôt que sur le succès individuel de celles qui réussissent les épreuves des examens.

\subsubsection{4 - Les universités, leur caractère social et un espace pour l'émancipation}

L'article de Lauren Erdreich et Tamar Rapoport (2002) sera le seul examiné dans cette section. Cette étude est une bonne illustration des recherches sociologiques féministes contemporaines en éducation qui postulent que les femmes, tout en vivant dans une société dont un certain nombre de ces institutions sont patriarcales, peuvent, malgré ces contraintes, acquérir du pouvoir sur leur propre parcours (Collectif Laure-Gaudreault, 1997; Bouchard et Cloutier, 1998; Fauzia, 2001). Un autre postulat de ces approches ${ }^{136}$ est que les institutions éducatives ont elles-mêmes un caractère social en sus de la reproduction des relations de pouvoir externes et qu'elles peuvent contribuer à produire (émanciper) de nouveaux groupes sociaux, pourvu toutefois qu'elles offrent la possibilité de développer une pensée critique. Cette étude reflète également la complexité des interrelations entre les diverses identités, dans ce cas ethnonational, pour des Palestiniennes vivant en Israël et étudiant à l'Université hébraïque à Jérusalem.

Erdreich et Rapoport ont réalisé une étude ${ }^{137}$ qualitative en vue de répondre aux deux questions suivantes : 1) De quelles manières s'établissent les liens entre l'identité ethnonationale et la connaissance construite dans les expériences d'appren-

133. " [...] horrific ordeals, test of character, and sacred masculine rituals » (Deslandes, $2002: 577)$.

134. " [...] to cast the successful victors in supremely masculine terms, much as they did in their descriptions of brawny athletes, scrappy soldiers, and plucky adventurers » (Deslandes, 2002: 578).

135. "In many ways, the need to bolster the masculine significance of examinations was not simply the product of the economic, social, and cultural changes that accompanied the rise of professional society. Rather, it sprang from the need to preserve male prerogatives at institutions that were frequently characterized as under siege by women who [...] appropriate, challenge, and occasionaly subvert the meaning of a process that Oxbridge men assumed was their alone » (Deslandes, 2002: 548).

136. Ce postulat est partagé également par le courant de la " nouvelle sociologie de l'éducation » au cours des années 80 : H. GIROUX (1983), "Theories of Reproduction and Resistance in the New Sociology of Education », Harvard Educational Review, 53, 3: 257-294, cité dans Erdreich et Rapoport (2002, 495); Dandurand et Ollivier (1987).

137. ERDREICH, Lauren et RAPOPORT, Tamar (2002). « Elaborating Ethnonational Awareness via Academic Literacy: Palestinian Israeli Women at the University », Anthropology and Education Quarterly, 33, 4, mai : 492-515 (article 3). 
tissage à l'université? et 2) Quelle est la signification selon le sexe (gendered meaning) de ces liens pour les femmes? Les données à la base de leur publication portent sur des entrevues en profondeur réalisées par Erdreich en 1998-1999 auprès des groupes suivants : a) onze jeunes femmes Palestiniennes Israéliennes étudiant à l'Université hébraïque à Jérusalem; $b$ ) la représentante étudiante arabe du comité étudiants-professeurs; $c$ la présidente de l'Association étudiante arabe; $d$ ) trois chargés de cours; et $e$ ) les six assistants de ces cours. Erdreich et Rapoport ont également fait de l'observation participante auprès de ces étudiantes dans leurs diverses activités parascolaires, y compris pour certaines dans leur famille. Ces chercheuses ont aussi profité du cours Introduction à la sociologie, en tant que "laboratoire» naturel, afin d'explorer la confrontation initiale d'un groupe marginal avec la connaissance universitaire culturelle structurée. Elles ont observé cette population étudiante en action dans ce cours durant trois années scolaires (1998-2001). Parmi les 240 étudiantes et étudiants, 30 seulement étaient des Palestiniennes Israéliennes et 3 des hommes du même groupe ethnonational ${ }^{138}$. Les étudiantes interrogées étaient inscrites à ce cours. La majorité était inscrite dans cette université à deux majeures ${ }^{139}$, dont la majeure en éducation. Sept en étaient à leur première année d'études universitaires, tandis que deux fréquentaient l'université pour la deuxième année.

Les transitions sont multiples et impliquent, selon les chercheuses, divers rites de passage pour ces jeunes filles du fait qu'elles entrent plus jeunes à l'université que les étudiantes juives et se retrouvent, pour la plupart, éloignées pour la première fois de leur ville et de leur famille, et ce, dans un contexte universitaire où elles sont minoritaires et marginalisées comme Palestiniennes, femmes ${ }^{140}$, chrétiennes ou musulmanes et comme jeunes adultes. Dans leur processus de scolarisation au secondaire, à l'intérieur d'un système scolaire où l'État d'Israël contrôle et supervise l'école publique arabe, ces jeunes femmes n'ont pas été habituées à mettre en doute l'autorité. Il en est de même dans leur groupe ethnonational par rapport au pouvoir des hommes (père et frères), ces derniers étant considérés, poursuivent les auteures, comme les gardiens traditionnels de l'autorité collective.

Ces diverses situations sociologiques "obligent» le groupe dominé à de plus grandes adaptations, et pour plusieurs à l'intégration au groupe dominant dans l'espoir d'acquérir pouvoir et statut. D'autres types de transition sont aussi à vivre pour ces jeunes femmes, notamment concernant la connaissance et le processus d'apprentissage à l'université. Par exemple, la mémorisation et la "redite des bonnes réponses » aux examens ne sont plus appropriées. Les enjeux sociaux et politiques se

138. Ces étudiantes et étudiants palestiniens israéliens étaient plus jeunes que leurs pairs juifs du fait que ces derniers avaient l'obligation, après avoir terminé leurs études secondaires, de faire un séjour de deux ans dans l'armée.

139. Pour satisfaire aux normes universitaires, les étudiantes et les étudiants doivent s'inscrire à deux programmes menant chacun à l'obtention d'une majeure.

140. Les auteures mentionnent la progression des femmes à l'université parmi leur groupe ethnonational. En 1984, les femmes constituaient $25 \%$ du groupe contre $44 \%$ en 1996: M. Al-Haj (1999) cité dans Erdreich et Rapoport (2002: 492). 
trouvent maintenant au cœur des processus de connaissance. On s'attend qu'elles formulent des critiques, les analysent et les appliquent à ces enjeux ${ }^{141}$.

Malgré les contradictions et coupures par rapport à leurs expériences antérieures scolaires et sociales, ces jeunes femmes vivent de nouvelles situations qui leur offrent aussi, selon les chercheuses, un espace pour des changements sociaux et politiques et, dans le cas présent, pour la construction, à travers cet accès à la connaissance et à la vie universitaire, d'un nouveau « discours » identitaire ethnonational et sexué.

Les chercheuses inscrivent leur recherche dans une approche critique sociologique poststructuraliste, dans laquelle il est postulé que les institutions éducatives ont elles-mêmes un caractère social en sus de la reproduction des relations de pouvoir externes ${ }^{142}$. Elles retiennent l'idée de Paul Gee (1990) que le processus de scolarisation (literacy) et la connaissance sont intégrés et favorisent la constitution d'un "discours", des pratiques sociales et des idées d'un groupe particulier (Erdreich et Rapoport, 2002: 492). Ciblant leur analyse sur les discours de jeunes femmes sur l'université et sur leurs familles, Erdreich et Rapoport montrent comment le processus de scolarisation, à travers le discours scolaire dominant, vise la reproduction ethnonationale et sexuée ainsi que les rapports de domination et aussi comment de jeunes femmes utilisent ces discours afin d'en reconstruire d'autres sur leur propre identité ethnonationale ${ }^{143}$ et sexuée ${ }^{144}$ (gender ${ }^{145}$ ). Un autre objectif poursuivi par les chercheuses est d'ordre épistémologique, soit l'illustration du pouvoir des significations extracurriculaires du processus de scolarisation ${ }^{146}$.

Erdreich et Rapoport ne partagent pas les hypothèses de chercheuses et de chercheurs aux yeux de qui l'établissement scolaire ou encore les enseignantes et les enseignants sont les seules personnes responsables, par des pratiques pédagogiques émancipatrices, de l'éveil et de la reconstruction de «discours » chez les individus de groupes dominés. Ces propositions nieraient, d'une part, la capacité des étudiantes et des étudiants dans ce processus et, d'autre part, l'effet de prise de conscience chez ces jeunes du fait de leur confrontation à différents discours, notamment à travers les cours. Les chercheuses classent d'ailleurs les cours en trois catégories : «the "Othering”

141. " The women must adjust to this new knowledge, ways of thinking, and the skills they demand. This disruption has both " technical" and subjective aspects. Primary ways of knowing-memorizing and regurgiting correct answers-are no longer appropriate. Social and political issues are now a major focus of discussion. Rather than obedience and submission, the women are expected to raise criticism, found it soundly, and apply it to social issues. Adjustment to this new way of learning forces them to reflect on isssues related to their place in social hierarchies » (Erdreich et Rapoport, 2002: 493).

142. "Educational institutions themselves have a social character beyond reproduction of external power relations »: Giroux (1983) cité dans Erdreich et Rapoport (2002: 495).

143. Il s'agit ici de leur identité de Palestiniennes Israéliennes par rapport aux Juifs vivant en Israël.

144. La variable du sexe est considérée par rapport à leurs frères du même groupe ethnonational.

145. « Because literacy for Palestinian Israëli women is acquired in a situation of subjugation, literacy in the dominant discourse can elaborate awareness of the means by which knowledge creates domination. When this discourse is itself reflexive it can also provide the tools to oppose domination. This opposition, however, is not always limited to one dominant versus non dominant axis, but is often also applied to internal power relations, such as gender » (Erdreich et Rapoport,2002: 511).

146. " For educational anthropologists, understanding how literacy facilitates inclusion of these women in the nondominant discourse of Palestinian ehtnonationalism exemplifies the power of extracurricular meanings of literacy » (Erdreich et Rapoport, 2002: 511). 
courses $^{147}$, the "Other's" view ${ }^{148}$, the Context-blind classes ${ }^{149}$ ». Elles montrent comment ces jeunes femmes arrivent, à travers un processus réflexif, à cette reconstruction ${ }^{150}$. Cette dernière suit plusieurs phases : c'est d'abord le déni des inégalités ou de leur situation de dominées, puis la prise de conscience des inégalités suivie de la valorisation de leur propre groupe ethnonational et, enfin, il y a construction d'un nouveau discours narratif. À travers ces réflexions et analyses, elles arrivent peu à peu à s'autoriser, comme femmes, à créer un discours.

Erdreich et Rapoport (2002) concluent leur étude en mentionnant que les effets non anticipés du processus de scolarisation en enseignement supérieur pour les groupes dominés devraient être analysés à la fois par rapport au groupe dominant, dans le cas présent ethnonational, et à l'intérieur du groupe dominé, dans ce cas-ci sexué. Elles indiquent en outre que leur étude qualitative contribue à l'avancement de la pédagogie féministe poststructuraliste ${ }^{151}$.

\subsubsection{5 - La socialisation sexuée et les programmes de sciences}

L'étude $^{152}$ canadienne de Valerie A. Haines et Jean E. Wallace (2002) s'inscrit dans le courant des études féministes qui centrent leur analyse sur le processus de socialisation sexuée et son impact sur l'inscription des jeunes femmes à des programmes d'études moins fréquentés traditionnellement par les femmes. Dans une enquête ${ }^{153}$ menée auprès d'un échantillon d'étudiantes et d'étudiants inscrits à une majeure ${ }^{154}$ en sciences (121) et à une majeure en sciences sociales $(160)^{155}$, dans une université située dans une ville de grande densité de l'Ouest canadien, les chercheuses tentent de déterminer si la socialisation sexuée (gender socialization) et ses produits - les rôles et les stéréotypes sexuels (gender roles and gendered stereotypes)-agissent comme intermédiaires dans l'inscription aux programmes de sciences ${ }^{156}$ des jeunes femmes et hommes. Trois groupes de facteurs constituent les

147. Le contenu de ces cours est alimenté par la société juive: "The "0thering" courses explain general concepts from within Jewish society » (Erdreich et Rapoport, 2002: 503).

148. Les cours exprimant les points de vue de la population palestinienne: "Classes from the view of the Palestinian Other » (Erdreich et Papoport, 2002: 504).

149. " [...] "neutral" one in which "Otherness" seemed irrelevant» (Erdreich et Papoport, 2002: 505).

150. Dans l'esprit des auteures, si aucun de ces cours n'avait pour objet, dans ses objectifs et pratiques, l'émancipation des groupes dominés, la confrontation des discours n'aurait toutefois pas la même portée.

151. " [...] by revealing how liberal participation in literacy can be an empowering tool in reconstructing conceptions of women in ethnonational discourses » (Erdreich et Rapoport, 2002: 492).

152. HAINES, Valerie A. et WALLACE, Jean E. (2002). "Exploring the Association of Sex and Majoring in Science ", The Alberta Journal of Educational Research, XLVIII, 2, été: 188-192 (article 4).

153. Un questionnaire, dont la majorité des questions était fermée (closed-ended), a servi à recueillir les données. Le test de la régression logistique a été utilisé dans le traitement des données.

154. C'est un programme de premier cycle (undergraduate).

155. Au total, $60 \%$ de l'échantillon est constitué de femmes et $40 \%$ d'hommes. La moyenne d'âge est de 24 ans.

156. L'appartenance sexuelle est la variable indépendante principale (femme $=1)$, tandis que l'inscription à une majeure en sciences est la variable dépendante (en sciences $=1$ ). 
variables témoins : $a$ ) les attitudes par rapport aux rôles sexuels ${ }^{157} ; b$ ) la préparation scolaire au secondaire en sciences; et $c$ ) les perceptions de soi et les expériences à l'université158.

Le fait d'être une femme (appartenance sociale sexuelle) est lié négativement à la fréquentation d'un programme de sciences. L'hypothèse principale formulée par les chercheuses se vérifie donc, et est statistiquement significative (modèle 1). Il en est de même des attitudes par rapport aux rôles sexuels lorsque ces variables sont prises en considération avec l'effet de l'appartenance sexuelle (modèle 2) : la relation initiale demeure significative, et des attitudes moins traditionnelles sont rattachées à une plus grande probabilité d'inscription à un programme de sciences. Étant donné cependant que ce groupe de facteurs ne réduit pas le coefficient associé à l'appartenance sexuelle, les auteures concluent à l'absence réelle de médiation de ces variables d'attitudes par rapport aux rôles sexuels. L'ajout aux deux ensembles précédents du groupe b) de facteurs (modèle 3) réduit de $40 \%$ le coefficient de l'appartenance sexuelle du modèle 2 et le rend non significatif. Par ailleurs, l'ajout aux trois ensembles précédents du groupe c) de facteurs (modèle 4) montre une relation significative qui va dans le sens des hypothèses des chercheuses ${ }^{159}$. Toutefois, ces facteurs ne modifient pas l'effet de la relation initiale, le coefficient augmentant légèrement.

Haines et Wallace concluent qu'il n'y a pas de relation significative entre le fait d'être une femme ou un homme (appartenance sexuelle) et la probabilité de s'inscrire à un programme de sciences (majeure). Elles ajoutent que, même si des attitudes moins traditionalistes par rapport à la famille et à l'emploi, un plus grand intérêt pour les sciences et un plus grand soutien afin de fréquenter un programme de sciences augmentent les probabilités de s'inscrire à ces programmes, aucun de ces facteurs n'a un effet de médiation sur la relation initiale (modèle 1). Seul le fait d'avoir suivi un cours poussé de mathématiques ${ }^{160}$ et un plus grand nombre de cours de sciences ${ }^{161}$ au secondaire modifie la relation initiale. Ces résultats justifient, selon les chercheuses, que des réformes soient apportées aux programmes d'études au secondaire et dans les pratiques pédagogiques afin d'augmenter le nombre de jeunes filles dans les cours de mathématiques et de sciences au secondaire.

157. Deux indicateurs ont été codifiés sur une échelle de 1 (désapprouve fortement) à 5 (fortement en accord): a) « Belief that a family and a top-level science career are compatible for women »; b) « Belief that society encourages women to pursue science » (Haines et Wallace, 2002: 190, tableau 1).

158. "University self perceptions and experiences "; trois indicateurs ont été codifiés sur une échelle de 1 à 5 : a) la perception de ses habiletés concernant les mathématiques; b) son niveau d'intérêt relativement à la science; c) la somme des encouragements reçus de divers groupes (père, mère, personnel enseignant du secondaire, corps professoral à l'université, etc.) à poursuivre des études en sciences.

159. Un score plus élevé sur chacun des indicateurs est lié à une plus grande fréquentation d'un programme de sciences.

160. Cet élément a été codifié 1 ou 0. Ces résultats corroborent ceux d’autres études: Chipman et Wilson (1985) et Farmer et al. (1995) cités dans Haines et Wallace (2002: 190).

161. Cela correspond au nombre de cours de chimie, de biologie et de physique suivis au secondaire (Haines et Wallace, 2002 : tableau 1). 


\subsubsection{6 - La famille et le soutien aux études}

Les études qui ont retenu de multiples rapports sociaux et leurs interrelations complexes (de race ou d'ethnie, de sexe ou de classe) ont montré l'influence du soutien et des stratégies des parents, en particulier des mères, dans la persévérance scolaire de leurs enfants (Dyhouse, 2002). L'importance de la famille est encore plus grande, selon Cheng et Starks (2002), pour la poursuite et la persévérance scolaire des jeunes des groupes victimes de racisme du fait de leurs expériences de discrimination dans la société et du besoin qui en découle de côtoyer des personnes significatives dans la famille. Ces études contribuent au développement de la recherche sur la réussite éducative par le fait qu'elles incorporent dans leur modèle d'analyse d'autres personnes significatives (closes relatives) dans un réseau familial élargi.

Marisela Rosas et Florence A. Hamrick (2002) ont fait de même dans leur étude $^{162}$, et leur apport est important en vue de la compréhension de la réussite éducative chez de jeunes Mexicaines-Américaines. J'ai classé cet article dans le cinquième groupe des recherches du fait que les auteures y accordaient plus d'importance à l'appartenance « raciale ou ethnique » qu'à celle de l'appartenance sexuelle.

Selon ces chercheuses, les Hispaniques, ou les Latinas et les Latinos, constituent le groupe minoritaire le plus nombreux aux États-Unis. Approximativement $8 \%$ des titulaires d'un diplôme du secondaire s'inscrivent au collège ou à l'université, mais $30 \%$ abandonnent leurs études secondaires ${ }^{163}$. Plus de $60 \%$ des étudiantes et des étudiants hispaniques sont inscrits dans des collèges communautaires (two-year college), même si leur nombre a triplé parmi la population étudiante de premier cycle aux États-Unis de 1976 à $1996^{164}$. Les femmes hispaniques sont plus nombreuses à poursuivre des études collégiales et universitaires que les hommes du même groupe ethnique ${ }^{165}$.

Rosas et Hamrick ont utilisé une approche phénoménologique dans leur étude afin de comprendre comment les répondantes ${ }^{166}$ décrivent et "construisent » leurs décisions de poursuivre des études postsecondaires. De plus, elles voulaient découvrir si et comment ces décisions reflétaient des normes, des croyances ou des prémisses (assumptions) du travail, caractéristiques de leur milieu social d'origine. Ces chercheuses formulent l'hypothèse, partagée par d'autres, que les définitions de la famille à l'intérieur de la culture mexicaine-américaine incorporent des réseaux

162. ROSAS, Marisela et HAMRICK, Florence A. (2002). « Postsecondary Enrollment and Academic Decision Making: Family Influences on Women College Students of Mexican Descent », Equity \& Excellence in Education, 35, 1, avril: 59-69 (article 11).

163. Dervarics (1997) cité dans Rosas et Hamrick (2002: 59).

164. The Nation (2001) cité dans Rosas et Hamrick (2002: 59).

165. En 1980, $51 \%$ de la population étudiante hispanique était composée de femmes; en 1999, cette proportion atteignait $57 \%$ : The Nation (2001) cité dans Rosas et Hamrick (2002: 59).

166. Sept femmes américaines de descendance mexicaine ont participé à cette étude. Elles fréquentent alors un établissement d'enseignement supérieur situé dans un État du Midwest américain, avec une concentration d'à peu près $2 \%$ d'Hispaniques pour $95 \%$ de Blancs. Ces jeunes femmes sont inscrites à divers types d'universités et programmes d'études. Six des sept participantes ont un ou une membre de leur famille immédiate qui a fréquenté ou fréquente le collège ou l'université. Trois ont des parents ou des grands-parents qui sont allés au collège. Les parents de six d'entre elles ont divorcé et elles vivent avec leur mère, chef de famille. Elles ont grandi dans le Midwest ou le Sud-Ouest des États-Unis. Toutefois, aucune de ces étudiantes ne réside depuis longtemps dans l'État où elles sont inscrites aux études au moment des entrevues. 
multiples qui englobent ceux de la famille immédiate (nucléaire) et de la famille étendue de même que ceux qui regroupent les comadres et compadres, c'est-à-dire des amies et des amis proches à travers l'institution sociale du compadrazgo ${ }^{167}$. L'appartenance à ces réseaux implique obligations et responsabilités, réciprocité et solidarité entre les membres. Rosas et Hamrick vérifient ces définitions élargies de la famille chez les participantes à l'étude. Elles constatent en outre que la famille nucléaire ou élargie (mère, frères et sœurs, tantes, comadres et compadres) a influencé ou stimulé principalement la première phase du processus décisionnel, soit la construction et la formation des intentions de poursuivre ses études collégiales. Le réseau de la famille des pairs de descendance mexicaine (corésidence à l'université, sororité, etc.) a été plus influent dans la troisième phase, soit celle de l'intégration, de la persévérance et de la réussite des études collégiales. Les chercheuses expliquent ce résultat par le fait que la famille immédiate n'est pas toujours en mesure de fournir l'aide nécessaire concernant l'apprentissage dans les cours et les façons les plus appropriées de se débrouiller dans les diverses interactions de la vie universitaire. Dans leurs discours, les jeunes femmes expriment également leurs obligations de réussite et leurs responsabilités symboliques et concrètes quant à leur famille ${ }^{168}$. Ce dernier point est corroboré sur le plan historique avec des jeunes Afro-Américaines (Bertaux et Anderson, 2001).

\section{3 - L'analyse et la discussion des résultats}

Le thème sur lequel porte mon étude est celui de la réussite éducative en enseignement postsecondaire examiné à travers l'ensemble du processus, soit de l'accès aux divers cheminements scolaires jusqu'à l'insertion professionnelle, en passant par la diplomation. Concernant l'analyse et la discussion des résultats des 29 études recensées, je demeure toutefois prudente dans la généralisation des résultats du fait que ces recherches ont été menées dans des pays qui n'ont pas les mêmes systèmes organisationnels d'enseignement postsecondaire, ces derniers ne s'étant pas développés de plus dans les mêmes contextes sociétaux. En outre, quelques-uns des travaux faisant l'objet de la présente recension ont été réalisés en recourant à une méthode qualitative, dans une optique de compréhension plus fine des phénomènes à l'étude et non de généralisation des résultats. Compte tenu de ces limites inhérentes à mon processus de recherche, je tente ci-dessous de dégager des tendances générales par rapport à l'hypothèse du maintien et de la progression des femmes par rapport à la réussite éducative aux études postsecondaires (accès, parcours scolaire et insertion professionnelle) au cours des années à venir dans les pays industrialisés tels les États-Unis, l'Angleterre et le Canada. À cette fin, je réfléchis principalement à

167. " The comadres and compadres [...] are formalized peer relationships that knit close friends into each other's families as extended family members. Compadrazgo relationships are assumed to be long-lasting, and have the same status as close relatives [...] " (Rosas et Hamrick, 2002: 59).

168. Le terme désigne ici l'ensemble des réseaux familiaux. 
partir des modèles théoriques et des résultats des 11 études classées dans le champ, ou plus près du champ des études féministes (groupes 1-5). J'appuie également ma démonstration sur des résultats des autres études (groupes 6-10) ainsi que sur d'autres travaux scientifiques menés au Québec et ailleurs.

Bien que les femmes soient majoritaires aux études collégiales et au premier cycle universitaire dans les pays industrialisés, il est possible d'observer à partir de la présente recension des écrits scientifiques (publiés en 2002) que le sexe est retenu comme variable indépendante ou témoin par 25 des 29 équipes de recherche (tableau 1). Par ailleurs, 4 équipes traitent exclusivement l'appartenance sexuelle dans leur analyse ${ }^{169}$. Cependant, la majorité des équipes incluent à leur modèle d'analyse d'autres types d'appartenances, les principales étant l'origine socioéconomique (18 articles) et la race ou l'ethnie (17 articles) ${ }^{170}$. Notons que l'inclusion dans les analyses féministes sociologiques de l'origine socioéconomique s'est faite rapidement et presque automatiquement, compte tenu de la formation acquise traditionnellement en sociologie. Par ailleurs, l'inclusion des appartenances raciales ou ethniques a été plus lente, et je dirais qu'elle a été revendiquée et «imposée», non sans raison d'ailleurs, par la militance et l'arrivée progressive des femmes de descendance africaine, mexicaine et asiatique parmi les professeures et les chercheuses universitaires et les chercheuses des appareils d'État aux États-Unis et en Angleterre notamment. Cette inclusion a été jugée nécessaire en vue de la compréhension des situations sociologiques et des conditions de vie des femmes. Une telle façon d'approcher les phénomènes s'est imposée au cours des années 80 dans les études de sciences sociales appliquées à l'éducation dans ces deux pays (Cloutier, 1990). D'ailleurs, l'apport de ces recherches est manifeste dans la présente recension des écrits scientifiques liés au domaine de l'éducation postsecondaire.

\section{1 - La progression des femmes concernant la réussite éducative aux études postsecondaires}

Les jeunes femmes sont mieux représentées dans les établissements d'enseignement postsecondaire dans les pays industrialisés depuis les années 80. Il y a moins de diversité entre les femmes de différentes appartenances socioéconomique et ethnique ou raciale comparativement aux jeunes hommes (Beattie, 2002). Dans ce dernier groupe, les hommes, «blancs", de classe moyenne-supérieure dominent et se distinguent plus massivement des autres par une poursuite et une persévérance plus élevées quant aux études postsecondaires (Beattie, 2002; Jacobs et Berkowitz, 2002). Les étudiantes, comme groupe social, ont donc progressé concernant leur inscription aux études postsecondaires durant les dernières décennies. Cependant,

169. Voir, à titre d'exemple, l'étude d'Aveling (2002) que j'ai classée dans les études féministes (groupe 1).

170. Le parent pauvre des appartenances sociales qui ont fait l'objet d'analyse dans ces écrits scientifiques est l'âge ou la problématique des étudiantes et des étudiants adultes. Dans les deux groupes d'études que j'ai classées comme des études féministes ou plus près de ces cadres d'analyses sociologiques, une seule recherche (Jacobs et Berkowitz King, 2002) regroupe, dans son échantillon, des étudiantes adultes. L'étude de Duraisamy (2002) et celle de Deslandes (2002) traitent l'âge en termes de cohorte ou de période historique. 
cette progression ne s'est pas réalisée dans tous les programmes d'études, notamment du côté des sciences (Haines, 2002; Ramirez et Wotipka, 2001) et en sciences appliquées (Gadalla, 2001). De plus, les femmes, comme les jeunes de milieu socioéconomique modeste et les jeunes afro-américains et hispaniques, fréquentent moins les institutions les plus sélectives et élitistes (Karen, 2002; Lang, 2002). Les jeunes femmes et jeunes hommes de minorités raciales ou ethniques s'inscrivent dans une plus faible proportion aux établissements menant aux études supérieures ${ }^{171}$. En outre, les femmes (à niveau de scolarité et diplômes similaires) ne retirent pas les mêmes bénéfices de leur scolarisation postsecondaire, des écarts salariaux s'observant entre les sexes à la faveur des hommes (Schwartz et Finnie, 2002).

Les facteurs avancés dans la compréhension d'une plus grande progression des femmes en éducation postsecondaire sont de divers types. Ainsi, les mouvements sociaux féministes et des droits civils, dans les pays industrialisés (Aveling, 2002; Borst, 2002; Solórzano et Yosso, 2002; Prendergast, 2002), malgré la difficulté d'en quantifier les effets, ont influé sur les États ${ }^{172}$ et les organismes internationaux dans la reconnaissance du droit à l'éducation pour toute personne sans distinction de son appartenance sociale et dans la mise en place de réformes pour atteindre les objectifs d' "égalité des chances ». Des systèmes économiques davantage prospères depuis la Seconde Guerre mondiale ont également favorisé le développement du capital humain par l'entremise des systèmes scolaires (Robert et Tondreau, 1997).

De leur côté, les mesures de redressement (affirmative action), particulièrement par rapport à la race ou à l'ethnie, ont joué favorablement pour augmenter le nombre de ces jeunes aux études supérieures aux États-Unis jusqu'à la fin des années $70^{173}$ (Borst, 2002; Solórzano et Yosso, 2002). Une marge d'autonomie des universités par rapport aux institutions de la société civile a aussi permis un décloisonnement social plus rapide dans ces établissements. Le système d'enseignement supérieur a "produit » volontairement, ou à son insu, de "nouveaux " groupes sociaux (Petitat, 1982), dont celui des femmes. Une plus grande prise en considération de la performance scolaire dans les politiques d'admission en enseignement supérieur a également favorisé un certain nombre de femmes (Larue, 1986). Les jeunes femmes ont fait preuve de moins de traditionalisme que les jeunes hommes en s'affranchissant plus systématiquement des stéréotypes sexuels, ce qui s'est concrétisé dans leur réussite scolaire (Bouchard et St-Amant, 1996), leurs aspirations scolaires et leur choix de programmes universitaires, plusieurs jeunes hommes s'excluant de programmes qu'ils jugent trop féminins ou féminisés. Le soutien des membres de la famille, en particulier des mères, et l'aide de réseaux plus larges que la famille nucléaire ou étendue chez des jeunes de minorités de descendance mexicaine (Rosas et Hamrick, 2002) ou africaine (Cheng et Starks, 2002) ont contribué à stimuler les jeunes femmes en vue de la poursuite de leurs études postsecondaires.

171. Sont ici visés le baccalauréat, la maîtrise et le doctorat.

172. Les États ont adopté, notamment, des chartes des droits.

173. J'aimerais cependant voir confirmer par des études, dont je ne dispose pas actuellement, si l'effet de ces programmes a été plus important pour les jeunes hommes des minorités ethniques que pour les jeunes femmes des mêmes groupes ethniques. 
Les jeunes femmes se distinguent moins entre elles selon la classe sociale, la race et les habiletés cognitives que les jeunes hommes par rapport à leur anticipation des retombées monétaires dans la décision de poursuivre des études postsecondaires. Ces attentes, même chez un grand nombre de jeunes hommes comme de jeunes femmes, ne sont toutefois pas les principaux motifs ou situations à l'origine de leur décision de poursuivre des études (Beattie, 2002). D'autres motifs s'ajoutent pour les jeunes femmes, telle la possibilité de réaliser l'ensemble de leurs projets de vie (mariage, enfants, carrière) et d'améliorer leur qualité de vie (mobilité sociale et capital culturel) (Aveling, 2002).

\section{2 - Le travail invisible et l'insertion professionnelle}

Selon les études considérées, les jeunes femmes australiennes, même lorsqu'elles sont élevées dans un contexte éducationnel d'égalité entre les sexes, ne peuvent concrétiser leurs aspirations de mener de front leurs projets de vie familiaux et professionnels, contrairement à leurs partenaires. Elles doivent réduire leurs aspirations et expériences professionnelles pour s'occuper de leurs enfants (Aveling, 2002). Les contradictions vécues par des femmes anglaises au cours des années 50 et 60 (Dyhouse, 2002) ne sont pas encore résolues pour les jeunes femmes de cohortes plus récentes. Leurs gains ou progressions, par rapport à leurs aînées, se situent du côté d'une plus grande acceptation de leurs rôles d'épouse et de travailleuse salariée, non encore toutefois par rapport à ceux de mère et de travailleuse salariée, le travail « invisible » contraignant davantage les jeunes femmes mères que les jeunes hommes pères dans leur processus d'insertion professionnelle (Cloutier, Trottier et Laforce, 1998; Fahmy et Veillette, 1997; Spain, Bédard et Paiement, 1997). Toutefois, je formule l'hypothèse suivante: les femmes des cohortes récentes, comme celles des cohortes anciennes, souhaitent concrétiser les connaissances et les compétences acquises lors de leurs études postsecondaires dans la poursuite d'une carrière (Aveling, 2002; Dyhouse, 2002; Fahmy et Veillette, 1997; Fahmy, 1992).

Pour sa part, l'étude de Jacobs et Berkowitz King (2002) portant sur un échantillon (1995) de jeunes femmes américaines et de femmes plus âgées (15-44 ans) a montré que les statuts matrimonial et maternel ne distinguaient pas les femmes inscrites à temps partiel qui terminent leurs études collégiales de celles qui ne les achèvent pas. Ce résultat constitue un certain espoir relativement au point que je formulais plus haut pour les femmes adultes, celles-ci étant plus nombreuses parmi la population étudiante inscrite à temps partiel. Toutefois, dans la même étude, les facteurs qui permettent de distinguer les deux groupes (terminant ou non leurs études collégiales) laissent percevoir que les «lourds » mécanismes inhérents aux exigences liées à la «logique scolaire" s'appliquent et favorisent les plus jeunes et celles qui n'ont pas la responsabilité d'enfants. Ces mécanismes défavorables sont des arrêts d'études entre la fin de leurs études secondaires et l'inscription aux études collégiales ainsi que l'occupation d'un emploi à temps plein. Les femmes chefs de famille, sans bourse ni aide financière substantielle, diminuent donc grandement leurs possibilités de réaliser leurs projets d'études postsecondaires. Être célibataire et ne pas avoir d'enfants ou en avoir peu sont des conditions qui augmentent en outre, pour les 
jeunes femmes comme pour les jeunes hommes, les probabilités de s'inscrire au collège (Beattie, 2002; Jacobs et Berkowitz King, 2002). La maternité et la possibilité de cumuler ces obligations et responsabilités avec les exigences de la poursuite d'études postsecondaires, ou avec les exigences du processus d'insertion professionnelle, sont encore des enjeux de taille pour les femmes, jeunes et plus âgées.

\section{3 - L'examen de I'hypothèse du maintien et de la progression des femmes concernant la réussite éducative aux études postsecondaires}

Peut-on, avec ce corpus de recherche, faire l'hypothèse du maintien ou de la progression des femmes par rapport à la réussite éducative aux études postsecondaires (accès, parcours scolaire et insertion professionnelle) au cours des années à venir? Les recherches historiques menées au XIX ${ }^{\mathrm{e}}$ siècle aux universités d'Oxford et de Cambridge (Deslandes, 2002), durant la première moitié du XXe siècle aux États-Unis dans les écoles de médecine (Borst, 2002), de même que les études réalisées au cours de la seconde moitié du $\mathrm{XX}^{\mathrm{e}}$ siècle sur l'analyse des politiques et décisions légales aux États-Unis au sujet de la mixité scolaire raciale (Solórzano et Yosso, 2002; Prendergast, 2002), montrent encore plus clairement que les systèmes d'enseignement supérieur sont stratifiés, hiérarchisés tant par rapport aux types d'établissement que par rapport aux programmes d'études offerts. Les systèmes d'enseignement postsecondaire des pays industrialisés se sont complexifiés, modifiés pour répondre à la demande sociale de «nouveaux " groupes sociaux. Certains auteurs et auteures qualifient ces changements, pour l'enseignement universitaire, d'un passage d'un système élitiste à l'université de masse. La majorité des études de sciences sociales montrent cependant que ces systèmes ont mis au point d'autres types d'institutions pour répondre à la demande et ont laissé plus ou moins intactes, en fait de provenance sociale, organisationnelle et curriculaire, les "vieilles » universités élitistes (Karen, 2002; Longden, 2002; Gellert, 1997). Dans ce contexte, obtenir une "place » ou réussir à s'inscrire à tel établissement élitiste ou à tel programme d'études ${ }^{174}$ était et demeure un enjeu, non seulement de maintien de ses acquis ou de mobilité sociale, mais également de distinction de classe, de race, d'ethnie et de $\operatorname{sexe}^{175}$ (Borst, 2002; Deslandes, 2002; Solórzano et Yosso, 2002; Prendergast, 2002; Lang, 2002).

Dans ce contexte de distinctions sociales, les progrès faits par certaines personnes de minorités raciales ou ethniques au cours des années 70 dans ces établissements et programmes élitistes sont menacés par la riposte des élites. La contre-mobilisation des élites traditionalistes («blanches», de classe supérieure et moyennesupérieure) s'est déjà fait sentir dans la baisse de la représentation des jeunes de minorités raciales ou ethniques inscrits aux études supérieures depuis les années 80 (Karen, 2002; Borst, 2002; Solórzano et Yosso, 2002; Prendergast, 2002). Les mécanismes de la contre-mobilisation sont puissants, comme il a été possible de l'observer dans ces études. Ils sont de l'ordre de la construction symbolique : «le racisme

174. C'est le cas notamment dans les facultés de médecine et de droit.

175. Dans un ouvrage sur l'expansion des établissements d'enseignement supérieur aux États-Unis, Randall Collins (1979) ajoute également les enjeux liés aux dénominations religieuses et à la prolifération de leurs croyances et pouvoirs. 
Cette situation de contre-mobilisation s'amplifie dans un contexte où les jeunes femmes réussissent en plus grand nombre que les jeunes hommes sur le plan scolaire. n'est pas un problème systémique de discrimination, la race, c'est une affaire personnelle, privée» (Solórzano et Yosso, 2002 : 163). Les «Blancs» (au pouvoir), comme dans le cas de la Californie, argumentent qu'ils sont devenus une «minorité numérique» et qu'ils ont besoin de protection (Solórzano et Yosso, 2002). Ces mécanismes sont aussi d'ordre structurel ${ }^{176}$ et ils agissent sur les pratiques (politiques d'admission (Borst, 2002), mesures de redressement (affirmative action), etc.).

Dans ce contexte, étant donné la «jeunesse historique » de la reconnaissance du droit des femmes aux études supérieures dans les pays industrialisés, leur légitimité est menacée. Les jeunes femmes de classe sociale supérieure pourront être mieux protégées ${ }^{177}$, mais celles d'autres origines, en particulier les femmes d'un milieu socioéconomique faible ou de minorités raciales ou ethniques, seront à risque. Elles n'auront accès, comme maintenant pour la plupart, qu'aux réseaux scolaires moins prestigieux et ne menant pas directement aux études supérieures. À noter que cette situation de contre-mobilisation s'amplifie dans un contexte où les jeunes femmes réussissent en plus grand nombre que les jeunes hommes sur le plan scolaire. Par ailleurs, les médias contribuent actuellement à construire socialement cette nonlégitimité (Bouchard, Boily et Proulx, 2003), comme le faisaient au siècle dernier les jeunes hommes étudiants à Oxbridge en utilisant le discours de l'usurpation: les jeunes femmes "assiègent "leur" établissement» (Borst, 2002).

Ainsi, les facteurs socioéconomiques, raciaux ou ethniques marquent encore profondément les destins scolaires des jeunes femmes, même s'ils distinguent moins les comportements des femmes de différentes appartenances sociales comparativement à ceux des jeunes hommes (Beattie, 2002; Karen, 2002). Les parents et les mères ne disposent pas tous et toutes des biens et des outils nécessaires pour soutenir de manière appropriée leurs filles tout au long du processus d'études postsecondaires selon la culture scolaire dominante (Rosas et Hamrick, 2002). Formuler des aspirations scolaires élevées pour ses enfants ne garantit pas d'avoir une plus grande influence sur eux, la force de l'autorité patriarcale et de la division sociale raciale contrecarrant la prise de décision de mères et de pères de minorités ethniques (Cheng et Starks, 2002).

À l'intérieur des systèmes d'enseignement postsecondaire, la diversité en fait de provenance sociale n'est pas très valorisée. Pourtant, des exemples de pratiques existent pour montrer que la mixité sexuelle (Bouchard et al., 2003), raciale ou ethnique (Gurin et al., 2002) favorise la réussite éducationnelle de l'ensemble des étudiantes et des étudiants (meilleure réussite scolaire, ouverture sur la diversité, participation citoyenne). La diversité des modèles et des pratiques pédagogiques valorisant la culture des groupes non traditionnels aux études postsecondaires n'est toujours pas courante. Cependant, encore ici les effets sont positifs et prometteurs (Hampton et Roy, 2002; Good et al., 2002). D’autres études ont montré que le «métier d'étudiant et

176. Cela ajoute grandement à leur pouvoir d'imposition.

177. Dans l'affaire Grutter v. Bollinger (1997) soumise à la Cour suprême des États-Unis, c'est une femme "blanche ", Barbara Grutter, qui est la plaignante, cette dernière ayant fait une demande sans succès à la Faculté de droit de l'Université du Michigan (Solórzano et Yosso, 2002). Il serait intéressant, aux fins de mes hypothèses, de disposer de l'information sur son origine sociale. 
d'étudiante s'apprend ", autant pour ce qui est de l'apprentissage de nouveaux rites de passage qu'en ce qui concerne les méthodes et les pratiques d'études (Boyer, Coridian et Erlich, 2001; Good et al., 2002).

Cette diversité implique cependant pour assurer son succès que l'on y consacre du temps et des ressources humaines. Dans le contexte présent du néolibéralisme, des compressions budgétaires sont faites dans le financement des établissements postsecondaires et dans l'aide financière aux études, et ce, dans de nombreux pays industrialisés (Kirby, 2002; Longden, 2002). L'idéologie néolibérale s'impose également peu à peu dans les pratiques des collèges et des universités (Giroux ${ }^{178}$, 2002; Quirke et Davies, 2002). Les établissements scolaires postsecondaires, même les établissements publics, prennent de moins en moins de risques par rapport au recrutement de leurs élèves. Et certaines universités (dont des universités canadiennes) modifient leurs pratiques, comme celles de hausser les frais d'inscription à des programmes d'études où l'admission est contingentée (Quirke et Davies, 2002). Les premières générations à poursuivre des études postsecondaires-femmes, jeunes de multiples appartenances sociales - et leurs familles sont davantage sensibles à ces hausses de financement et contraintes par celles-ci, ce qui peut compromettre leurs décisions de poursuivre leurs projets d'études postsecondaires ou de persévérer dans ceux-ci (Fenske, Porter et Dubrock, 2001).

Compte tenu de ces divers éléments, je demeure donc prudente quant à la formulation sans condition de l'hypothèse du maintien ou de la progression des femmes concernant la réussite éducative aux études postsecondaires dans les pays industrialisés au cours des années à venir, et ce, encore plus pour les femmes adultes et les jeunes femmes des milieux sociaux moins favorisés.

\section{Conclusion}

\section{L'éducation postsecondaire pour les femmes: espace d'émancipation et de transformations sociales}

Les féministes en éducation, en particulier au cours des années 70 et 80 au Québec comme dans d'autres pays industrialisés, ont été très critiques à l'égard des systèmes scolaires. Elles leur reprochaient de niveler et de refouler les ambitions et les aspirations scolaires et professionnelles des jeunes filles et de contribuer à reproduire la division sociale sexuelle du travail. De nombreux travaux de féministes dans les universités, appareils d'États, syndicats et groupes de femmes ont contribué à cet éveil critique et à ces analyses (Bouchard, Cloutier et Hamel, 1996). Plusieurs féministes résistaient toutefois à la pensée que les principales solutions aux rapports sociaux de sexe ne viendraient que de la transformation des mentalités (valeurs moins sexistes dans les manuels scolaires, par exemple), ou d'une présence massive des jeunes femmes dans des programmes d'études traditionnellement choisis par les

178. Henry Giroux (2002) utilise le concept de « capitalisme académique ». 
jeunes hommes. À leur avis, les changements devaient être plus profonds et se faire dans l'ensemble des programmes d'études et des pratiques organisationnelles et pédagogiques, de manière à former des jeunes filles et des jeunes hommes, différents, égaux, en vue de transformations sociales plus fondamentales (Cloutier, Mura et Parent, 1987). Par la suite, devant la lenteur des réformes et des progrès anticipés, plusieurs ont contribué à créer des cours féministes dans différents programmes, principalement du côté des sciences sociales et de l'histoire, pour ensuite mettre sur pied des programmes d'études féministes (Doré, 1997). Malgré les luttes constantes qu'il a fallu mener à l'intérieur des universités pour créer et développer ces programmes, ceux-ci ont eu des effets non seulement sur les étudiantes et les quelques étudiants qui les ont fréquentés mais aussi sur les établissements, par leur effet mobilisateur en fait de militance et de recherche. La parole, les «discours» et les expériences des femmes ont été rendus plus visibles, ce que Dyhouse (2002) a exprimé d'ailleurs dans la poursuite de ses recherches. Les féministes avaient, et ont toujours, comme projet, tel que l'écrit Nado Aveling (2002 : 265), de libérer les femmes « de pratiques et de structures » qui ont perpétué leur position inégale dans la société (Fahmy et Bouchard, 1997). Leurs recherches et actions ont eu des retombées positives sur les mères et leurs filles et sur la poursuite de leurs études et la persévérance scolaire de ces dernières (Bouchard et al., 2003).

Bien que plusieurs changements anticipés ne se soient pas matérialisés dans le domaine de l'éducation et dans d'autres secteurs de la société, les féministes en sciences sociales appliquées à l'éducation ont poursuivi sans cesse leurs recherches et actions en mettant encore davantage l'accent sur la dimension d'émancipation, sur la fonction d'empowerment des études supérieures pour les jeunes femmes malgré les contradictions et les imperfections des établissements d'enseignement postsecondaire ${ }^{179}$. L'étude d'Erdreich et Rapoport (2002) de même que celle de Fauzia (2001) sont de bons exemples en ce sens. Malgré ces contradictions, ces jeunes femmes ont pu, grâce au processus de connaissance propre au milieu universitaire et à la présence d'espaces critiques mobilisateurs, arriver peu à peu à construire et à créer leurs propres « discours» identitaires, ethnonational par rapport au groupe dominant dans le cas des jeunes Palestiniennes Israéliennes, et sexué au sein de leur propre groupe national par rapport au pouvoir des hommes (père et frères).

À l'instar de Claudia, de Ruby Puentes et du juge Thurgood Marshall (Solórzano et Yosso, 2002; Fahmy et Bouchard, 1997) je ne peux m'empêcher de rappeler en terminant la nécessité de l'éducation dans la prise de conscience des inégalités et du développement des habiletés d'empowerment, et ce, principalement pour les étudiantes et les étudiants qui sont les premières personnes de leurs milieux respectifs à fréquenter le collège ou l'université. Pour ma part, je continuerai avec d'autres partageant le même projet à maintenir et à élargir ces espaces d'émancipation en enseignement postsecondaire!

179. Plusieurs perçoivent toutefois la contre-mobilisation par rapport à la réussite plus grande des femmes en enseignement supérieur comme menaçante pour la légitimité de leur présence et leur maintien (Bouchard, Boily et Proulx, 2003). 


\section{Références bibliographiques 180}

BELLEMARE, Isabelle (2003). Regards des sciences sociales sur l'accès à l'enseignement postsecondaire, les cheminements, la réussite éducative et l'insertion professionnelle. Recension d'écrits scientifiques II, essai de maîtrise en sciences de l'orientation, Québec, Département des fondements et des pratiques en éducation, Faculté des sciences de l'éducation, Université Laval. (À paraître en ligne: Centre de recherche interuniversitaire sur l'éducation et la vie au travail [www.fse.ulaval.ca/crievat].)

BERTAUX, Nancy E., et M. Christine ANDERSON (2001). «An Emerging Tradition of Educational Achievement: African American Women in College and the Professions, 1920-1950 », Equity \& Excellence in Education, 34, 2, 16-21.

BOUCHARD, Pierrette, et Renée CLOUTIER (1998). «Présentation», Recherches féministes, Éducation et émancipation, 11, 1, 1-6.

BOUCHARD, Pierrette, et Jean-Claude ST-AMANT (1996). Garçons et filles, stéréotypes et réussite scolaire, Montréal, Les éditions du remue-ménage.

BOUCHARD, Pierrette, Isabelle BOILY et Marie-Claude PROULX (2003). La réussite scolaire comparée selon le sexe: catalyseur des discours masculinistes, Ottawa, Condition féminine Canada / Status of Women Canada, Recherche en matières de politiques / Policy Research.

BOUCHARD, Pierrette, en collaboration avec Renée CLOUTIER et Thérèse HAMEL (1996). «La recherche en éducation», dans Huguette Dagenais (dir.), Science, conscience et action, vingt-cinq ans de recherche féministe au Québec, Montréal, Les éditions du remue-ménage, 149-182.

BOUCHARD, Pierrette, Jean-Claude ST-AMANT, Nathalie RINFRET, Claudine BAUDOUX et Natacha BOUCHARD (2003). Les héritières du féminisme, t. II, Québec, Chaire d'étude Claire-Bonenfant sur la condition des femmes, Université Laval.

BOYER, Régine, Charles CORIDIAN et Valérie ERLICH (2001). «L'entrée dans la vie étudiante. Socialisation et apprentissages ", Revue française de pédagogie, 136, juillet-août-septembre, 97-105.

CLOUTIER, Renée (1990). «Savoirs féministes : création et transmission », communication présentée au Colloque interuniversitaire : l'Université "avec » les femmes, Université de Montréal, 25 octobre.

CLOUTIER, Renée, Claude TROTTIER et Louise LAFORCE (1998). «Les projets de vie et l'insertion professionnelle de femmes et d'hommes titulaires d'un baccalauréat ", Recherches féministes, Éducation et émancipation, 11, 1, 111-132.

180. À noter que certains des articles répertoriés dans l'annexe II ont aussi été mentionnés dans cet article. Je ne les indique pas toutefois dans la présente section mais dans l'annexe II. 
CLOUTIER, Renée, Roberta MURA et France PARENT (1987). « Tendances et perspectives de la recherche sur les femmes et l'éducation au Canada, 19751986 », dans Recherche et progrès en éducation, Bilan et prospective, Actes du Premier Congrès des sciences de l'éducation de langue française du Canada, 13-15 mai, 49-54.

CLOUTIER, Renée, Isabelle BELLEMARE, Isabelle CÔTÉ, et Hélène PARÉ (à paraître 2005a). «Regards des sciences sociales 2001 sur l'accès à l'enseignement postsecondaire, les cheminements, l'insertion socioprofessionnelle et la réussite éducative», dans Pierre Chenard et Pierre Doray (dir.), L'enjeu de la réussite dans l'enseignement supérieur, Sainte-Foy, Les Presses de l'Université du Québec.

CLOUTIER, Renée, Isabelle BELLEMARE, Isabelle CÔTÉ, et Hélène PARÉ (à paraître 2005b). «Regards des sciences sociales 2001 sur l'accès à l'enseignement postsecondaire, les cheminements, l'insertion professionnelle et la réussite éducative. Construction d'une typologie ", Notes de recherche du CIRST/CAPRES, Centre interuniversitaire de recherche sur la science et la technologie / Consortium d'animation pour la persévérance et la réussite dans l'enseignement supérieur.

COLLECTIF LAURE-GAUDREAULT (1997). Femmes, éducation et transformations sociales, Montréal, Les éditions du remue-ménage.

COLLINS, Randall (1979). The Credential Society. An Historical Sociology of Education and Stratification, New York, Academic Press.

CÔTÉ, Isabelle (2003). Regards des sciences sociales sur l'accès à l'enseignement postsecondaire, les cheminements, la réussite éducative et l'insertion professionnelle. Recension d'écrits scientifiques III, essai de maîtrise en sciences de l'orientation, Québec, Département des fondements et des pratiques en éducation, Faculté des sciences de l'éducation, Université Laval. (À paraître en ligne: Centre de recherche interuniversitaire sur l'éducation et la vie au travail [www.fse.ulaval.ca/crievat].)

DANDURAND, Pierre, et Roland OUELLET (1990). Les grandes orientations de la recherche en sociologie de l'éducation au Québec: un bilan bibliographique, Les Cahiers du LABRAPS, Sainte-Foy, Laboratoire de recherches en administration et politique scolaires, Faculté des sciences de l'éducation, Université Laval, série Études et documents, vol. 6.

DANDURAND, Pierre, et Émile OLLIVIER (1987). «Les paradigmes perdus. Essai sur la sociologie de l'éducation et son objet», Sociologie et sociétés, XIX, 2, 87-101.

DAUNE-RICHARD, Anne-Marie, et Anne-Marie DEVREUX (1992). « Rapports sociaux de sexe et conceptualisation sociologique», Recherches féministes, $5,2,7-30$. 
DAVID, Miriam E., Stephen, J. BALL, Jackie DAVIES et Diane REAY (2003). « Gender Issues in Parental Involment in Student Choices of Higher Education», Gender and Education, 15, 1, 21-37.

DELPHY, Christine (1998). L'ennemi principal, t. 1 : «Économie politique du patriarcat », Paris, Éditions Syllepse, Collection Nouvelles Questions féministes.

DELPHY (DUPONT), Christine (1970). «L'ennemi principal », Partisans, 54, 5, 157-172.

DESCARRIES, Francine (1998). «Le projet féministe à l'aube du XXI ${ }^{\mathrm{e}}$ siècle : un projet de libération et de solidarité qui fait toujours sens ", Cahiers de recherche sociologique, 30, 179-210.

DESCARRIES-BÉLANGER, Francine, et Shirley ROY (1988). Le mouvement des femmes et ses courants de pensée : essai de typologie, Les documents de l'ICREF 19, Ottawa, Institut canadien de recherches sur les femmes.

DORÉ, C. (1997). «Les études féministes : quelques observations sur la documentation au tournant des années 1990 », dans Collectif Laure-Gaudreault, Femmes, éducation et transformations sociales, Montréal, Les éditions du remueménage, 155-193.

FAHMY, Pauline, et Pierrette BOUCHARD (1997). «L'éducation féministe», dans Collectif Laure-Gaudreault, Femmes, éducation et transformations sociales, Montréal, Les éditions du remue-ménage, 31-53.

FAHMY, Pauline, et Diane VEILLETTE (1997). «Les femmes et le monde du travail rémunéré : l'indispensable conciliation entre vie et carrière ", dans Collectif Laure-Gaudreault, Femmes, éducation et transformations sociales, Montréal, Les éditions du remue-ménage, 247-295.

FAHMY, Pauline (1992). Femmes, entre vie et carrière. Le difficile équilibre, Montréal, Les Éditions Adage inc.

FAUZIA, Ahmad (2001). «Modern Traditions? British Muslim Women and Academic Achievement», Gender and Education, 13, 2, 137-152.

FAVE-BONNET, Marie-Françoise, et Nicole CLERC (2001). « Des « Héritiers » aux "nouveaux » étudiants : 35 ans de recherches », Revue française de pédagogie, 136, juillet-août-septembre, 9-19.

FENSKE, Robert H., John D. PORTER et Caryl P. DUBROCK (2000). « Tracking Financial Aid and Persistence of Women, Minority, and Needy Students in Science, Engineering, and Mathematics ", Research in Higher Education, $41,1,67-94$.

GADALLA, Tahany M. (2001). «Patterns of Women's Enrolment in University Mathematics, Engineering and Computer Science in Canada, 1972-1995 ", The Canadian Journal of Higher Education / La Revue canadienne d'enseignement supérieur, 31, 1, 1-34. 
GELLERT, Claudius (1997). «Les élites contre l'enseignement supérieur de masse. Une dichotomie mal conçue », Enseignement supérieur en Europe, XXII, 2, 109-118.

LARUE, Andrée (1986). L'accès des femmes aux savoirs rentables, mémoire de maîtrise en éducation (administration et politique scolaires), Québec, Faculté des études supérieures, Université Laval.

JUTEAU, Danielle, et Nicole LAURIN (1988). «L'évolution des formes de l'appropriation des femmes: des religieuses aux "mères porteuses" ", Revue canadienne de sociologie et d'anthropologie, 25, 2, 183-207.

MARRY, Catherine (2001). «Filles et garçons à l'école : du discours aux controverses des années 1990 », dans Jacqueline Laufer, Catherine Marry et Margaret Maruani, Masculin-féminin: questions pour les sciences de l'homme, Paris, Presses universitaires de France, Collection Sciences sociales et sociétés, 25-41.

MOSCONI, Nicole (1995). "Femmes et rapport au savoir», dans Pierrette Bouchard (dir.), Femmes et savoir. Les Cahiers de recherche du GREMF, 69, 7-30.

PARÉ, Hélène (2003). Regards des sciences sociales sur l'accès à l'enseignement postsecondaire, les cheminements, la réussite éducative et l'insertion professionnelle. Recension d'écrits scientifiques I, essai de maîtrise en sciences de l'orientation, Québec, Département des fondements et des pratiques en éducation, Faculté des sciences de l'éducation, Université Laval. (À paraître en ligne: Centre de recherche interuniversitaire sur l'éducation et la vie au travail [www.fse.ulaval.ca/crievat].)

PETITAT, André (1982). Production de l'école, production de la société, Analyse sociohistorique de quelques moments décisifs de l'évolution scolaire en Occident, Genève, Droz.

RAMIREZ, Francisco O., et Christine Min WOTIPKA (2001). «The Global Expansion of Women's Participation in Science and Engineering Fields of Study, 1972-1992 », Sociology of Education, 74, juillet, 231-251.

ROBERT, Marcel, et Jacques TONDREAU (1997). L'école québécoise, Débats, enjeux et pratiques sociales. Une analyse sociale de l'éducation pour la formation des maîtres, Montréal, Les éditions CEC inc.

SPAIN, Armelle, Lucille BÉDARD et Lucie PAIEMENT (1998). «Conception révisée du développement de carrière au féminin », Recherches féministes, Éducation et émancipation, 11,1, 95-109. 


\section{Annexe I : Les revues répertoriées}

\section{Les revues ayant fait l'objet de la recension}

\section{Les revues sélectionnées}

Anthropoly and Education Quarterly (2002*)

Economics of Education Review

Equity \& Excellence in Education

Gender and Education

Harvard Educational Review (2002)

History of Education Quarterly (2002)

La Revue canadienne d'enseignement supérieur / The Canadian Journal of Higher Education

The Alberta Journal of Educational Research

Oxford Review of Education (2002)

Research Papers in Education. Policy and Practice

Scandinavian Journal of Educational Research (2002)

Sociology of Education

\section{Les revues examinées et non sélectionnées**}

Comparative Education Review

Curriculum Inquiry (2002***)

Educational Administration Quarterly

Educational Research and Evaluation (2002)

Educational Review

Educational Studies

Educational Theory

International Journal of Educational Development (2002)

Leadership and Policy Journal (2002)

Les Sciences de l'éducation pour l'ère nouvelle, revue internationale

* Articles répertoriés dans cette revue, uniquement pour l'année 2002, et non dans la recension précédente en 2001.

*: Revues ne comportant pas d'articles sur les thèmes de la recension.

*.: Revues examinées uniquement pour l'année 2002, et non dans la recension précédente. 
Planning and Changing. An Education Leadership and Policy Journal (2002)

Politiques et gestion de l'enseignement supérieur / Higher Education Management and Policy (2002)

RES Academica (2002)

Research in Education (2002)

Review of Educational Research

Revue suisse des sciences de l'éducation (2002)

The Journal of Experimental Education (2002)

\section{Annexe II : Les articles répertoriés et la catégorisation selon les cadres d'analyse}

\section{Groupe 1 : Le cadre d'analyse est féministe}

1. AVELING, Nado (2002). " "Having It All” and the Discourse of Equal Opportunity: Reflections on Choices and Changing Perceptions ", Gender and Education, 14, 3, 265-280.

2. DYHOUSE, Carol (2002). «Graduates, Mothers and Graduate Mothers: Family Investment in Higher Education in Twentieth-Century England ", Gender and Education, 14, 7, 325-336.

3. ERDREICH, Lauren, et Tamar RAPOPORT (2002). « Elaborating Ethnonational Awareness via Academic Literacy: Palestinian Israeli Women at the University ", Anthropology and Education Quarterly, 33, 4, mai, 492-515.

4. HAINES, Valerie A., et Jean E. WALLACE (2002). «Exploring the Association of Sex and Majoring in Science», The Alberta Journal of Educational Research, XLVIII, 2, été, 188-192.

\section{Groupe 2 : Un des cadres d'analyse est féministe}

5. BORST, Charlotte G. (2002). «Choosing the Student Body: Masculinity, Culture, and the Crisis of Medical School Admissions, 1920-1950 ", History of Education Quarterly, 42, 2, été, 181-214.

\section{Groupe 3 : Un des cadres d'analyse s'inscrit dans le courant des inégalités sociales liées aux rapports sociaux de sexe}

6. BEATTIE, Irenee R. (2002). «Are All "Adolescent Econometricians" Created Equal? Racial, Class, and Gender Differences in College Enrollment », Sociology of Education, 75, 1, janvier, 19-43. 
7. DESLANDES, Paul R. (2002). "Competitive Examinations and the Culture of Masculinity in Oxbridge Undergraduate Life, 1850-1920», History of Education Quarterly, 42, 4, hiver, 544-578.

8. DURAISAMY, P. (2002). « Changes in Returns to Education in India, 1983-94: By Gender, Age-cohort and Location », Economics of Education Review, 21, 6, 609-622.

9. JACOBS, Jerry A., et Rosalind BERKOWITZ KING (2002). «Age and College Completion : A Life-History Analysis of Women Aged 15-44», Sociology of Education, 75, 3, juillet, 211-230.

Groupe 4 : Un des cadres d'analyse est féministe et l'analyse porte principalement sur l'appartenance raciale ou ethnique

10. SOLÓRZANO, Daniel G., et Tara J. YOSSO (2002). «A Critical Race Counterstory of Race, Racism, and Affirmative Action », Equity \& Excellence in Education, 35, 2, mai, 155-168.

Groupe 5 : Un des cadres d'analyse s'inscrit dans le courant des inégalités sociales liées aux rapports sociaux de sexe et l'analyse porte principalement sur l'appartenance raciale ou ethnique

11. ROSAS, Marisela, et Florence A. HAMRICK (2002). « Postsecondary Enrollment and Academic Decision Making: Family Influences on Women College Students of Mexican Descent », Equity \& Excellence in Education, 35, 1, avril, 59-69.

Groupe 6 : Un des cadres d'analyse s'inscrit dans le courant des inégalités sociales liées aux appartenances sociales, dont l'appartenance sexuelle

12. CHENG, Simon, et Brian STARKS (2002). "Racial Differences in the Effects of Significant Others on Students' Educational Expectations », Sociology of Education, 75, 4, octobre, 306-327.

13. GURIN, Patricia, Eric L. DEY, Sylvia HURTADO et Gerald GURIN (2002). «Diversity and Higher Education: Theory and Impact on Educational Outcomes ", Harvard Educational Review, 72, 3, automne, 330-366.

14. JACOB, Brian A. (2002). «Where the Boys Aren't : Non-cognitive Skills, Returns to School and the Gender Gap in Higher Education ", Economics of Education Review, 21, 6, 589-598.

15. KAREN, David (2002). "Changes in Access to Higher Education in the United States : 1980-1992», Sociology of Education, 75, 3, juillet, 191-210. 
16. KILBRIDE MURPHY, Kenise, et Lucy D’ARCANGELO (2002). « Meeting Immigrant Community College Students' Needs on One Greater Toronto Area College Campus ", The Canadian Journal of Higher Education / La Revue canadienne d'enseignement supérieur, XXVII, 2, 1-26.

17. LANG, Katherine E. (2002). " "Flags and Slots" : Special Interest Groups and Selective Admissions", The Canadian Journal of Higher Education / La Revue canadienne d'enseignement supérieur, XXVII, 2, 103-142.

18. MARSH, Herbert W., et Sabina KLEITMAN (2002). «Extracurricular School Activities : The Good, the Bad, and the Nonlinear ", Harvard Educational Review, 72, 4, hiver, 464-514.

19. QUIRKE, Linda, et Scott DAVIES (2002). «The New Entrepreneurship in Higher Education: The Impact of Tuition Increases at an Ontario University ", The Canadian Journal of Higher Education / La Revue canadienne d'enseignement supérieur, XXVII, 3, 85-109.

20. SURTEES, P.G., N.W.J. WAINWRIGHT et P.D.P. PHAROAH (2002). «Psychosocial Factors and Sex Differences in High Academic Attainment at Cambridge University ", Oxford Review of Education, 28, 1, 21-38.

\section{Groupe 7 : Le sexe est considéré comme une variable indépendante et comme une variable témoin}

21. SCHWARTZ, S., et R. FINNIE (2002). «Student Loans in Canada: An Analysis of Borrowing and Repayment », Economics of Education Review, 21, 5, 497-512.

\section{Groupe 8 : Le sexe est considéré comme une des variables témoins}

22. SACHS, John (2002). "A Path Model for Students' Attitude to Writing a Thesis ", Scandinavian Journal of Educational Research, 46, 1, 99-108.

\section{Groupe 9 : Le sexe est considéré comme une des variables témoins, sans analyse particulière}

23. DEIL-AMEN, Regina, et James E. ROSENBAUM (2002). «The Unintended Consequences of Stigma-free Remediation ", Sociology of Education, 75, 3, juillet, 249-268.

24. HAMPTON, Mary, et Joan ROY (2002). «Strategies for Facilitating Success of First Nations Students », The Canadian Journal of Higher Education / La Revue canadienne d'enseignement supérieur, XXVII, 3, 1-28.

25. LONGDEN, Bernard (2002). «Retention Rates - Renewed Interest but Whose Interest Is being Served? », Research Papers in Education, 17, 1, 3-29. 


\section{Groupe 10 : Le sexe n'a pas été retenu comme variable parmi les appartenances sociales}

26. GIROUX, Henry A. (2002). « Neoliberalism, Corporate Culture, and the Promise of Higher Education : The University as a Democratic Public Sphere», Harvard Educational Review, 72, 4, hiver, 425-463.

27. GOOD, Jennifer M., Glennelle HALPIN, et Gerald HALPIN (2002). «Enhancing and Evaluating Mathematical and Scientific Problem-Solving Skills of African American College Freshmen ", Equity \& Excellence in Education, 35, 1, avril, 50-58.

28. KIRBY, Dale (2002). «Statistics and the Canada Millennium Scholarship Foundation", The Canadian Journal of Higher Education / La Revue canadienne d'enseignement supérieur, XXVII, 3, 111-118.

29. PRENDERGAST, Catherine (2002). «The Economy of Literacy: How the Supreme Court Stalled the Civil Rights Movement », Harvard Educational Review, 72, 2, été : 206-229. 